\title{
Comparative transcriptomic analysis of the different developmental stages of ovary in red swamp crayfish Procambarus clarkii
}

Yizhi Zhong ${ }^{1}$, Wenbin Zhao', Zhangsheng Tang ${ }^{1}$, Liming Huang ${ }^{1}$, Xiangxing Zhu², Xiang Liang ${ }^{3}$, Aifen Yan², Zhifa Lu', Yanling Yu', Dongsheng Tang ${ }^{2}$, Dapeng Wang ${ }^{1 *}$ and Zhuanling $\mathrm{Lu}^{\mathrm{H}^{*}}$

\begin{abstract}
Background: The red swamp crayfish Procambarus clarkii is a freshwater species that possesses high adaptability, environmental tolerance, and fecundity. P. clarkii is artificially farmed on a large scale in China. However, the molecular mechanisms of ovarian development in $P$. clarkii remain largely unknown. In this study, we identified four stages of $P$. clarkii ovary development, the previtellogenic stage (stage I), early vitellogenic stage (stage II), middle vitellogenic stage (stage III), and mature stage (stage IV) and compared the transcriptomics among these four stages through next-generation sequencing (NGS).

Results: The total numbers of clean reads of the four stages ranged from 42,013,648 to 62,220,956. A total of 216, 444 unigenes were obtained, and the GC content of most unigenes was slightly less than the AT content. Principal Component Analysis (PCA) and Anosim analysis demonstrated that the grouping of these four stages was feasible, and each stage could be distinguished from the others. In the expression pattern analysis, 2301 genes were continuously increase from stage I to stage IV, and 2660 genes were sharply decrease at stage IV compared to stages I-III. By comparing each of the stages at the same time, four clusters of differentially expressed genes (DEGs) were found to be uniquely highly expressed in stage I (136 genes), stage II (43 genes), stage III-IV (49 genes), and stage IV (22 genes), thus exhibiting developmental stage specificity. Moreover, in comparisons between adjacent stages, the number of DEGs between stage III and IV was the highest. GO enrichment analysis demonstrated that nutrient reservoir activity was highest at stage II and that this played a foreshadowing role in ovarian development, and the GO terms of cell, intracellular and organelle participated in the ovary maturation during later stages. In addition, KEGG pathway analysis revealed that the early development of the ovary was mainly associated with the PI3K-Akt signaling pathway and focal adhesion; the middle developmental period was related to apoptosis, lysine biosynthesis, and the NF-kappa B signaling pathway; the late developmental period was involved with the cell cycle and the p53 signaling pathway.
\end{abstract}

Conclusion: These transcriptomic data provide insights into the molecular mechanisms of ovarian development in P. clarkii. The results will be helpful for improving the reproduction and development of this aquatic species.

Keywords: Different developmental stages, Molecular mechanisms, Ovary, Procambarus clarkii, Transcriptomics

\footnotetext{
* Correspondence: oucwdp@163.com; nicky.004@163.com

'Guangxi Academy of Fishery Sciences/Guangxi Key Laboratory of Aquatic

Genetic Breeding and Healthy Aquaculture, Nanning 530021, China

Full list of author information is available at the end of the article
}

(c) The Author(s). 2021 Open Access This article is licensed under a Creative Commons Attribution 4.0 International License, which permits use, sharing, adaptation, distribution and reproduction in any medium or format, as long as you give appropriate credit to the original author(s) and the source, provide a link to the Creative Commons licence, and indicate if changes were made. The images or other third party material in this article are included in the article's Creative Commons licence, unless indicated otherwise in a credit line to the material. If material is not included in the article's Creative Commons licence and your intended use is not permitted by statutory regulation or exceeds the permitted use, you will need to obtain permission directly from the copyright holder. To view a copy of this licence, visit http://creativecommons.org/licenses/by/4.0/ The Creative Commons Public Domain Dedication waiver (http://creativecommons.org/publicdomain/zero/1.0/) applies to the data made available in this article, unless otherwise stated in a credit line to the data. 


\section{Background}

The red swamp crayfish Procambarus clarkii originated in south-central America and northeastern Mexico [1]. The freshwater crayfish is an invasive species now widely distributed in Europe, Africa, and Asia [2-5]. P. clarkii was first introduced into Nanjing, China, from Japan in 1929 [6], and at present it can be found in freshwater habitats such as rivers, swamps, sloughs, and paddy fields [5]. Although P. clarkii could lead to economic losses and declines biodiversity [7], the crayfish is one of the most important aquaculture resources [7-9], since it is welcomed by a vast number of consumers for its delicious taste and high meat quality. As a successful invasive species, $P$. clarkii has advantageous traits including a short life cycle, high fecundity, and high disease resistance $[5,6]$. The species is highly adaptable and can disperse widely in the habitat and tolerate diverse environmental conditions $[4,10]$. Furthermore, P. clarkii has retained high levels of genetic diversity in both wild populations $[5,6,9,11,12]$ and commercial populations [13]; this contributes to avoiding the harmful effects of inbreeding, for adapting to different environments [14], and in the selection of good breeding germplasm for crayfish artificial culture [5]. At present, P. clarkii farming has become an important industry in China, with production reaching 1,638,700 tons and a total output value of 369 billion China Yuan (CNY) in 2018 [15]. Some successful reproductive results for commercial $P$. clarkii have been reported from areas such as Qianjiang, Hubei province, where breeding grounds of $P$. clarkii in China, extend over 200 ha and enclose a variety of artificial ponds [16]. The red swamp crayfish-rice culture is the major model for production of $P$. clarkii in China, a farming scheme that not only makes significant improvements in rural livelihood and food security but also contributes to eco-environmental benefits and sustainable development [17].

As a model organism, $P$. clarkii is not only used to investigate invasive routes and dispersal patterns $[11,18]$, but also to perform research on animal behavior [19-21] and environmental stress and toxicity [22-25]. With the rapid development of the aquaculture industry, $P$. clarkii is often infected by various pathogens such as bacteria, viruses, and spiroplasmas [26-29], resulting in severe decreases in $P$. clarkii production. Therefore, significant anti-microbial research and studies of the immune response of $P$. clarkii have been performed, especially using transcriptome analysis by next-generation sequencing (NGS) [29-32]. NGS is a high-throughput sequencing technology and constitutes a variety of strategies that depend on a combination of template preparation, sequencing and imaging, and genome alignment and assembly methods $[33,34]$. Compared with the traditional Sanger sequencing technology, the NGS can produce an enormous volume of sequence data at an unprecedented level of sensitivity and accuracy, in shorter times, and at a much cheaper cost $[33,35]$. Combined with the de novo assembly methods such as Trinity, the full-length transcriptome assembly from NGS data can be implemented without a reference genome and does not require the correct alignment of reads to known splice sites [35, 36]. Currently, NGS is frequently used to analyze the transcriptome variation of $P$. clarkii in a variety of research areas, including pathogen infection as mentioned above [26-29], the immune system [31, 37], neurohormone regulation [38], and gonadal development [37, 39].The NGS transcriptomic analysis of $P$. clarkii revealed that the ovary and testis, the major reproductive organs, were more closely related to the pathways of DNA replication, cell cycle, and meiosisyeast compared to other non-reproductive organs (e.g., hepatopancreas and muscle) [37]. In addition, using 454 pyrosequencing technology, a differential expression analysis between the sexually mature ovary and testis of $P$. clarkii was performed, and the results identified gonadal development related genes that were highly expressed in ovary and testis [39]. However, we still have a limited understanding of the gonadal development of $P$. clarkii. In order to promote the development of the $P$ clarkii industry and build a comprehensive breeding system, the developmental mechanisms of male and female $P$. clarkii should be further investigated.

In female $P$. clarkii, oocyte development is classified into seven stages according to morphological characteristics. These are oogonial, immature, avitellogenic, early vitellogenic, midvitellogenic, late vitellogenic, and postvitellogenic-resorptive stages. Except for the oogonial stage, the remaining stages could occur mature oocytes $[40,41]$. Ovarian maturation includes an increase in size as the oocytes proliferate and increase in diameter during yolk and lipovitellin uptake [40, 42]. Hence, based on the size and color of the ovary, ovarian development of $P$. clarkii can be separated into five stages in the order of non-developed ovary (transparent), undifferentiated ovary (white), poorly developed ovary (yellow), developed ovary (orange), and mature ovary (brown) [41, 43]. To date, some researchers have investigated factors that impact ovarian maturation in $P$. clarkii, including chemical compounds, steroids, and herbicides. Treatment of methylfarnesoate (MF) for different time periods could stimulate and enhance the ovarian maturation of $P$. clarkii [42], and MF alone or in combination with $17 \beta$-estradiol (but not in combination with JHIII (juvenile hormone III) or $17 \alpha$-hydroxyprogesterone) could improve oocyte growth through stimulating the synthesis of vitellin in the ovary [44]. However, 17 $\alpha$ hydroxyprogesterone could significantly increase the gonadosomatic index (GSI) and directly stimulate 
vitellogenin production in $P$. clarkii [45], indicating that $17 \alpha$-hydroxyprogesterone was in competition with MF in the ovary or that it was involved in a negative feedback loop [44]. Interestingly, the ovary was the main target organ for selenium (Se) accumulation, and an appropriate concentration of Se in the diet could remarkably improve the spawning rate and promote synchronized ovulation of P. clarkii [46]. Moreover, Atrazine, a widely used herbicide, could reduce vitellogenin content in the ovary and decrease the oocyte size in P. clarkii [47], so the crayfish-rice culture system should consider the effect of Atrazine on the reproductive performance of the crayfish.

At present, the proteomic comparison between previtellogenic and vitellogenic ovaries of $P$. clarkii is performed using two-dimensional gel electrophoresis and mass spectrometry [48], but the information obtained has been sparse, with only 22 differentially expressed proteins being identified. More recently, the transcriptome information from ovaries at stage IV of $P$. clarkii has expanded our understanding of ovarian development and embryogenesis and demonstrated that pcRDH11 may play an essential role in this aspect [49]. However, the molecular mechanisms of the ovarian developmental process in $P$. clarkii remain poorly understood, and this hinders our understanding of reproduction and thereby affects the artificial breeding industry of $P$. clarkii. Herein, we selected the final four stages of ovarian development of $P$. clarkii from the five stages reported in the previous studies [41, 43], the previtellogenic stage (undifferentiated ovary, stage I), the early vitellogenic stage (poorly developed ovary, stage II), the middle vitellogenic stage (developed ovary, stage III), and the mature stage (mature ovary, stage IV) to perform transcriptome comparisons between different stages through NGS. The results will provide insights into the molecular mechanisms of ovarian development of $P$. clarkii.

\section{Materials and methods}

\section{Ovarian tissue collection and identification of different} developmental stages

The $P$. clarkii used in this study were cultured at a commercial farm in Laibin $\left(23^{\circ} 39^{\prime} 92^{\prime \prime} \mathrm{N}, 109^{\circ} 23^{\prime} 34^{\prime \prime} \mathrm{E}\right)$, Guangxi, China, that has adopted the crayfish-rice culture pattern. Crayfish were captured monthly from May to September, 2019 using cylindrical traps, and the female crayfish were transferred to water tanks with adequate aeration at $28{ }^{\circ} \mathrm{C}$ for three days. The ovaries were collected and photographed to record their morphology and color, then immediately immersed in RNA preservation buffer (\#R0118, Beyotime, China), frozen in liquid nitrogen, and stored at $-80^{\circ} \mathrm{C}$ until use.

The development of the ovary was separated into four stages according to the morphology and color, as the previtellogenic stage (stage I, yellowish white), the early vitellogenic stage (stage II, yellow), the middle vitellogenic stage (stage III, dark orange or light brown), and the mature stage (stage IV, dark brown or black). To confirm the classification by histology, a portion of each ovary tissues was selected for HE staining. Finally, three samples of each stage were identified, defined as stage I (I_7, I_19 and I_20), stage II (II_17, II_27 and II_30), stage III (III_33, III_49 and III_52), and stage IV (IV_35, IV_36 and IV_37).

\section{RNA extraction, cDNA library construction, and Illumina sequencing}

The total RNA of each ovary sample was extracted using Total RNA Extractor (Trizol) (B511311, Sangon, Shanghai, China) according to the manufacturer's instructions. A Qubit RNA HS Assay Kit (Q32855, Invitrogen, Carlsbad, CA, USA) was used to detect the sample RNA concentration using a Qubit Fluorometer (Q32866, Invitrogen, Carlsbad, CA, USA). Agarose gel electrophoresis was used to detect RNA integrity and genomic DNA contamination. The RNA-seq cDNA library of $P$. clarkii ovary was constructed based on the polyA structure of mRNA at the 3'-terminus according to the Hieff NGS MaxUp Dual-mode mRNA Library Prep Kit for Illumina (12301ES96, YEASEN, Shanghai, China) comprising mRNA isolation and preparation, fragmentation, double strand cDNA synthesis, cDNA end repairment and dAtailing, DNA adapter ligation, and cDNA library amplification by PCR. Purification and fragment size screening of the cDNA library was performed using Hieff NGS DNA Selection Beads (12601ES56, YEASEN, Shanghai, China). The cDNA of the final library was verified by electrophoresis; fragments ranged in size from 300 to 500 base pairs (bp). Finally, the cDNA library was sequenced on an Illumina HiSeq 2500 instrument by Sangon Biotech (Shanghai, China).

\section{De novo assembly, clustering, and functional annotation}

The raw image data files generated by the Illumina HiSeq 2500 instrument were analyzed and converted into raw reads by CASAVA Base Calling. The quality of raw reads was visually evaluated by FastQC software version 0.11.2. The sequence adapters and low quality bases (Quality score $<20$ ) were filtered out, and short length reads $(<35 \mathrm{nt})$ were removed by Trimmomatic software version 0.36 [50] to obtain clean data. Then, the de novo clean data were assembled into transcripts by Trinity software version 2.4.0 [51], where the parameter min kmer_cov was set equal to 2, and other parameters were set to default values. The assembled transcripts were deredundant using RSeQC software version 2.6.1 [52], and the longest transcript in each transcript cluster was 
taken as a unigene reference sequence for subsequent analysis.

Gene functional annotations were separately performed according to the following databases: NT (NCBI nucleotide sequences, http://ncbi.nlm.nih.gov/), NR (NCBI nonredundant protein sequences, http://ncbi.nlm.nih.gov/), COG/KOG (Clusters of Orthologous Groups of proteins/ euKaryotic Ortholog Groups, https://www.ncbi.nlm.nih. gov/COG/) [53], Swiss-Prot (A manually annotated and reviewed protein sequence database), TrEMBL, PFAM (Protein family, http://pfam.xfam.org/) [54], CDD (Conserved Domain Database, https://www.ncbi.nlm.nih.gov/ cdd/) [55], GO (Gene Ontology, http://www.geeontology. org), and KEGG (Kyoto Encyclopedia of Genes and Genomes, http://www.kegg.jp) [56]. The annotations of NR, NT, CDD, COG/KOG, Swiss-Prot, TrEMBL, and PFAM were executed by NCBI Blast+ [57]. The GO annotation was harvested based on the results of Swiss-Prot and TrEMBL protein annotation according to the information from Uniprot (http://www.uniprot.org/) [58]. KEGG annotation was performed by KAAS (KEGG Automatic Annotation Server) version 2.1 [59].

\section{Analysis of differential expression and gene enrichment} Firstly, for sequence evaluation of RNA-seq, Bowtie2 software version 2.3.2 [60] was used to compare effective data of the samples to the transcripts obtained by splicing and to gather statistical mapping information. The duplicate reads and inserted fragment distribution were analyzed by RSeQC software version 2.6.1 [52]. Distribution of gene coverage statistics were performed using BEDTool software version 2.26.0 [61]. Secondly, for analysis of gene expression levels, Salmon software version 0.8.2 [62] and the WGCNA (weighted gene coexpression network analysis) $\mathrm{R}$ package version 1.51 [63] were used to calculate the gene expression quantity and to perform gene co-expression analysis, respectively. The comparative analysis of samples and other statistical analyses and exploration in multiple directions were processed based on the expression matrix of the samples. In order to make gene expression levels estimated between different genes and different experiments comparable, we introduced the concept of transcripts per kilobase of exon model per million mapped reads (TPM) to represent the abundance of a transcript and the gene expression level. The formula for TPM was as follows:

$$
\begin{aligned}
& T P M_{i}=\frac{X_{i}}{L_{i}} * \frac{1}{\sum_{j} \frac{X_{j}}{L_{j}}} * 10^{6} \\
& X_{i}=\text { total exon fragment } / \text { reads } L_{i}=\frac{\text { exon length }}{K B}
\end{aligned}
$$

Thirdly, for analysis of differential gene expression, DESeq2 R package version 1.12.4 [64] was utilized to acquire the differentially expressed genes (DEGs) according to the default parameters. The screening conditions were qValue $<0.05$ and $\mid$ Fold Change $\mid>2$ to visualize the results of differential expression model. The DEGs were mapped to the STRING protein-protein interaction network database (http://string-db.org/) [65] for protein interaction network construction. Then, based on the results of differential gene analysis, a Venn diagram and heat map were drawn, and a cluster analysis was carried out. Finally, for gene enrichment analysis, topGO R package version 2.24.0 [66] was used for analysis of GO enrichment, and the clusterProfiler $\mathrm{R}$ package version 3.0.5 [67] was used for KEGG pathway and KOG category enrichment analysis, then draw the associated analysis network diagram.

\section{Real-time quantitative PCR (RT-qPCR) for DEGs validation} In order to validate the expression profile of DEGs from RNA-seq, we chose nine DEGs for further RT-qPCR detection. One microgram of total RNA from each sample was reverse transcribed into cDNA by Maxima Reverse Transcriptase (EP0743, ThermoFisher Scientific, USA) according to the manufacturer's instructions. The RTqPCR was conducted in a final volume of $20 \mu \mathrm{L}$ that consisted of $10 \mu \mathrm{L}$ of SYBR Green PCR Master Mix (\#4309155, ThermoFisher Scientific, USA), $0.4 \mu \mathrm{L}$ of forward primer $(10 \mu \mathrm{M}), 0.4 \mu \mathrm{L}$ of reverse primer $(10 \mu \mathrm{M})$, $2 \mu \mathrm{L}$ of cDNA template and $7.2 \mu \mathrm{L}$ of $\mathrm{dd}_{2} \mathrm{O}$. The RTqPCR was performed in an ABI StepOne plus instrument (ABI, California, USA). The reaction conditions were as follows: denaturation at $95^{\circ} \mathrm{C}$ for $10 \mathrm{~min}$; followed by 45 cycles of $95^{\circ} \mathrm{C}$ for $15 \mathrm{~s}, 60^{\circ} \mathrm{C}$ for $30 \mathrm{~s}$; the melt curve was read according to instrument guidelines. Glyceraldehyde-3-phosphate dehydrogenase (GAPDH) was used as an internal reference gene for normalization. All primers used for RT-qPCR are shown in Supplementary Table S1. The fold changes of target genes between each comparison group were calculated according to the relative quantitative $2^{-\Delta \Delta \mathrm{Ct}}$ method formula. The RTqPCR experiment was performed triplicate, and the data were shown as mean \pm S.D. The statistical analysis was carried out based on one-way ANOVA.

\section{Results}

Identification of different ovarian developmental stages

The female crayfish were captured monthly from May to September, and the ovaries were collected and photographed to record the morphology and color (Fig. 1a-d). According to the color and size, we divided the ovaries into four stages, the previtellogenic stage (stage I), the early vitellogenic stage (stage II), the middle vitellogenic stage (stage III), and the mature stage (stage IV). The 

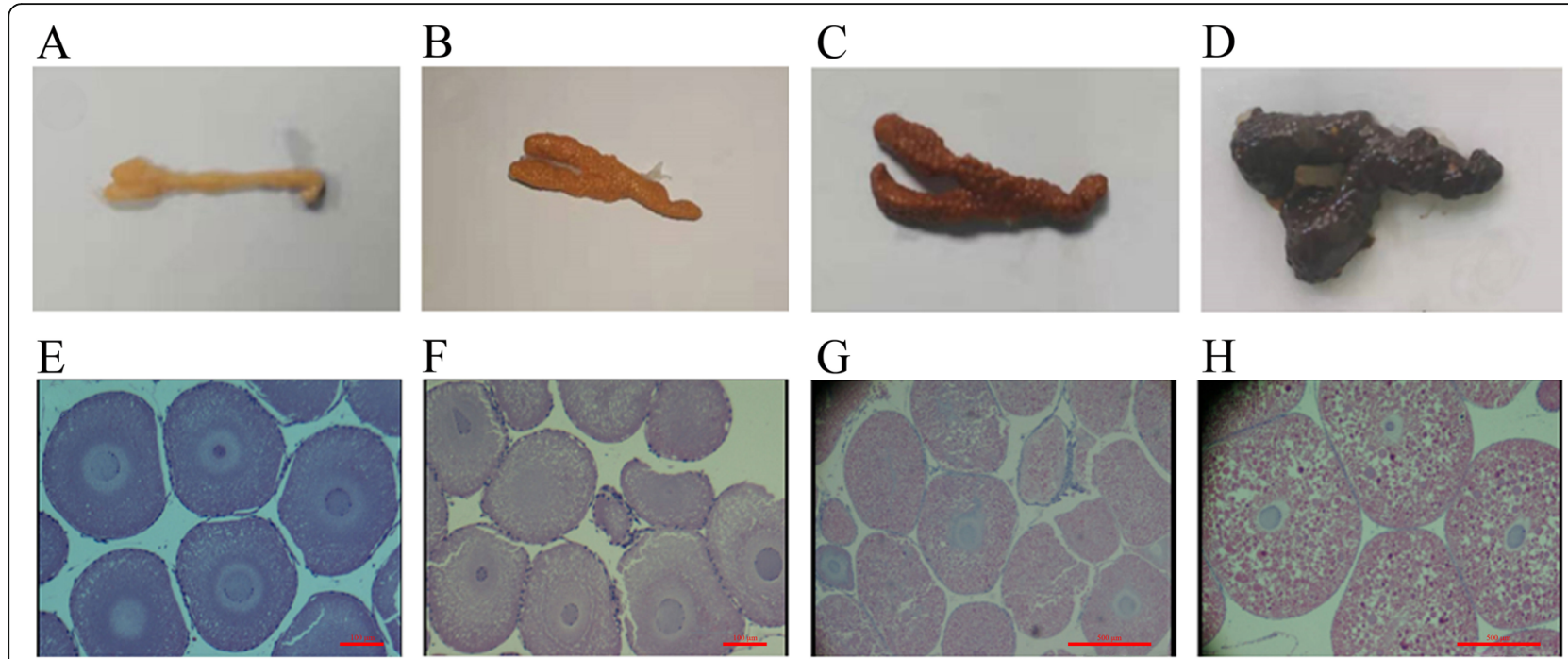

Fig. 1 Identification of ovaries at different developmental stages of P. clarkii. a-d: The morphology and color of ovaries at different stages by photograph; a: The morphology and color of the ovary at stage I, b: The morphology and color of the ovary at stage II, c: The morphology and color of the ovary at stage III, $\mathbf{d}$ : The morphology and color of the ovary at stage IV. E-H: The histomorphology of ovaries at different stages by HE staining; e: The oocytes at stage I, bar $=100 \mu \mathrm{m}$, f: The oocytes at stage II, bar $=100 \mu \mathrm{m}, \mathbf{g}$ : The oocytes at stage III, bar $=500 \mu \mathrm{m}, \mathbf{h}$ : The oocytes at stage IV, bar $=500 \mu \mathrm{m}$

stage I ovary was yellowish white and thin, the ovary outer membrane was thick, and the egg particles were inconspicuous (Fig. 1a); the stage II ovary was yellow and became larger, and the outer membrane became thinner, the egg particles were obvious and were the size of rice grains (Fig. 1b); the stage III ovary was light brown and larger than the stage II ovary, the size of egg particles continuously increased, and the particles were closely arranged (Fig. 1c); the stage IV ovary was dark brown or black, and the volume was extremely inflated, the eggs were plump and discrete (Fig. 1d). In addition, we detected the histomorphology of the ovary by HE staining to confirm the four stages (Fig. 1e-h). The oocytes at stage I were small and roundish, the diameter was $100-200 \mu \mathrm{m}$, and the nucleus and cytoplasm were blue by HE staining (Fig. $1 \mathrm{e})$; most of the oocytes at stage II were oval or subrotund and $200-500 \mu \mathrm{m}$ in diameter, the cytoplasm was stained in most red and partially blue (Fig. 1f), indicating that yolk granules began appearing; most of the oocytes at stage III were more than $500 \mu \mathrm{m}$ in diameter, the cytoplasm was obviously red by HE staining, and the number of yolk granules increased and the size became larger (Fig. 1g); The oocytes at stage IV were the largest $(>1000 \mu \mathrm{m})$ and were full of yolk granules that were larger than those at other stages and were dark red (Fig. 1h). These results revealed that the sizes of the oocytes and yolk granules increased as the ovary developed.

\section{Assembly and information analysis of transcriptome data} There were 12 ovary samples of $P$. clarkii in four different developmental stages that were subjected to RNAseq, including stage I (I_7, I_19 and I_20, named group A), stage II (II_17, II_27 and II_30, named group B), stage III (III_33, III_49 and III_52, named group C), and stage IV (IV_35, IV_36 and IV_37, named group D). Analyses were done in triplicate for each stage. The total raw read counts of all 12 samples ranged from 43,433, 438 to $64,090,726$ (Supplementary Table S2). The GC base ratios of raw data were between 45.31 and $51.71 \%$, with an average of was $47.2 \%$. Except for the sample IV $36(51.71 \%)$, the GC contents of the remaining 11 samples were less than 50\% (Supplementary Table S2 and Supplementary Figure S1), indicating that the GC ratio of the transcripts was less than the AT ratio in the ovary of $P$. clarkii. After quality control by removing adapters and low-quality bases (Quality score $<20$ ), the total clean read counts of all 12 samples ranged from 42,013,648 to $62,220,956$, while the total clean base counts ranged from 6,118,532,265 bp to 9,054,802,655 bp, and the Q30 base ratios (the proportion of nucleotides with quality value $\geq 30$ ) were $94.52-95.58 \%$. The GC base ratios were $45.08-51.35 \%$, and the average was $46.99 \%$ (Supplementary Table S3), consistent with the raw read data.

There were 445,326 transcripts and 216,444 unigenes obtained after assembly, and the N50 lengths were 1858 bp and $912 \mathrm{bp}$, respectively (Table 1). All of the transcripts and unigenes ranged from $201 \mathrm{bp}$ to $20,027 \mathrm{bp}$, 
Table 1 The assembly result of transcript and unigene

\begin{tabular}{llllllllll}
\hline & No. & $\mathbf{2 5 0 0}$ bp & $\mathbf{2 1 0 0 0}$ bp & $\mathbf{N 5 0}$ (bp) & $\mathbf{N 9 0}$ (bp) & Max Length (bp) & Min Length (bp) & Total Length (bp) & Average Length (bp) \\
\hline Transcript & 445,326 & 168,661 & 98,221 & 1858 & 292 & 20,027 & 201 & $381,942,933$ & 857.67 \\
Unigene & 216,444 & 62,141 & 26,934 & 912 & 253 & 20,027 & 201 & $133,770,495$ & 618.04
\end{tabular}

N50/N90: The length at 50\%/90\% of total length of the assembly transcript, which was the length of the cumulative transcript in the order from large length to small length

and there were 62,141 and 26,934 unigenes that were $\geq$ $500 \mathrm{bp}$ and $\geq 1000 \mathrm{bp}$, respectively (Table 1 ). The length distribution of the sequences showed that most of the transcripts and unigenes were less than $1000 \mathrm{bp}$ (Supplementary Figure S2A and Fig. 2a and b), accounting for 77.94 and $87.56 \%$, respectively. The GC content distribution demonstrated that the GC ratios of most transcripts and unigenes were less than 50\% (Supplementary Figure S2C and Fig. 2c) being mainly distributed around 40\%, coinciding with the raw and clean read data (Supplementary Table S2 and Supplementary Table S3). The isoform (also referred to as the transcript) number of each unigene indicated that $72.91 \%$ of the unigenes had only one isoform, and $11.66,4.24,2.58,1.63$, and $1.26 \%$ of the unigenes had 2, 3, 4, 5, and 6 isoforms, respectively (Fig. 2d).

\section{Overall functional annotation and analysis}

All 216,444 unigenes of the $P$. clarkii ovary were searched in the nine public databases NT, NR, KOG, Swiss-Prot, TrEMBL, PFAM, CDD, GO, and KEGG using a cut-off E-value of $10^{-5}$. There were $8872(4.1 \%)$, 23,683 (10.94\%), 10,520 (4.86\%), 14,913 (6.89\%), 25,706 (11.88\%), 8504 (3.93\%), 12,005 (4.86\%), 19,773 (9.14\%),

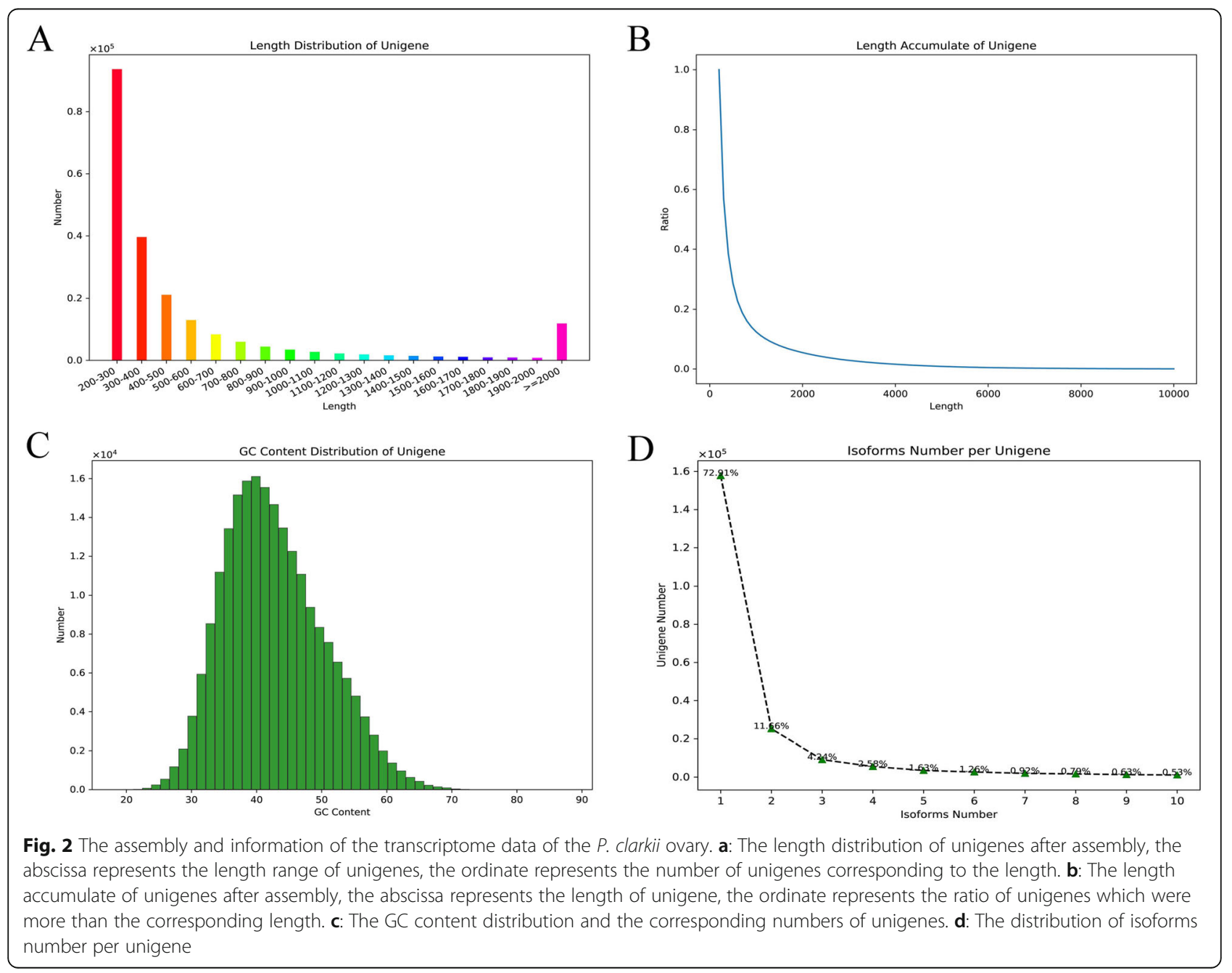


Table 2 The summary of gene annotation

\begin{tabular}{lll}
\hline Database & Number of genes & Percentage (\%) \\
\hline Annotated in NT & 8872 & 4.1 \\
Annotated in NR & 23,683 & 10.94 \\
Annotated in KOG & 10,520 & 4.86 \\
Annotated in Swissprot & 14,913 & 6.89 \\
Annotated in TrEMBL & 25,706 & 11.88 \\
Annotated in PFAM & 8504 & 3.93 \\
Annotated in CDD & 12,005 & 5.55 \\
Annotated in GO & 19,773 & 9.14 \\
Annotated in KEGG & 3560 & 1.64 \\
Annotated in at least one database & 32,599 & 15.06 \\
Annotated in all database & 1065 & 0.49 \\
Total genes & 216,444 & 100 \\
\hline
\end{tabular}

and $3560(1.64 \%)$ annotations, respectively (Table 2$)$. Of these, 32,599 (15.06\%) and $1065(0.49 \%)$ unigenes were annotated in at least one database and annotated in all databases, respectively (Table 2), indicating that most of the unigenes were not annotated. By comparison with the NR database, the transcript similarity between $P$. clarkii and similar species and the functional information of the homologous transcripts could be obtained (Supplementary Table S4). In the NR blast result, 1521 unigenes from the RNA-seq data in this study were best matched with the genes of Zootermopsis nevadensis, followed by Hydra vulgaris (1103 unigenes) and Limulus
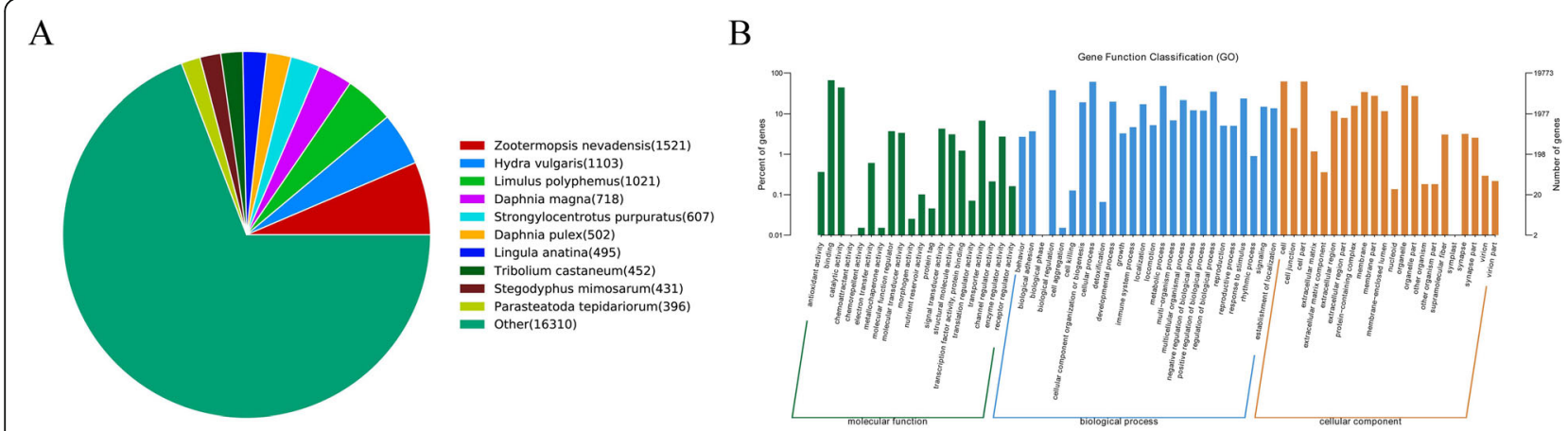

$\mathrm{C}$

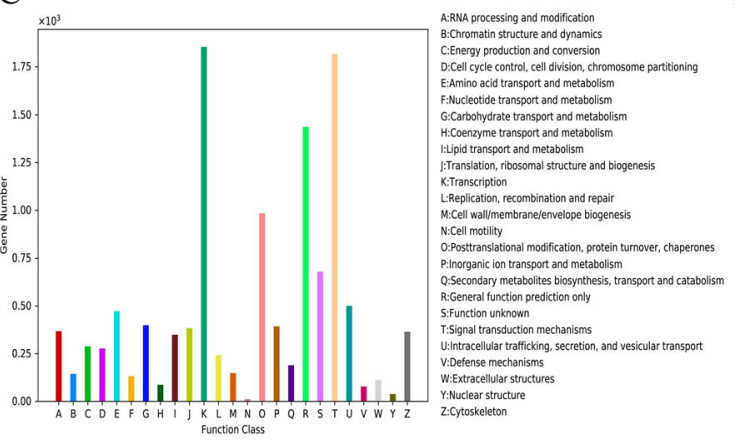

D

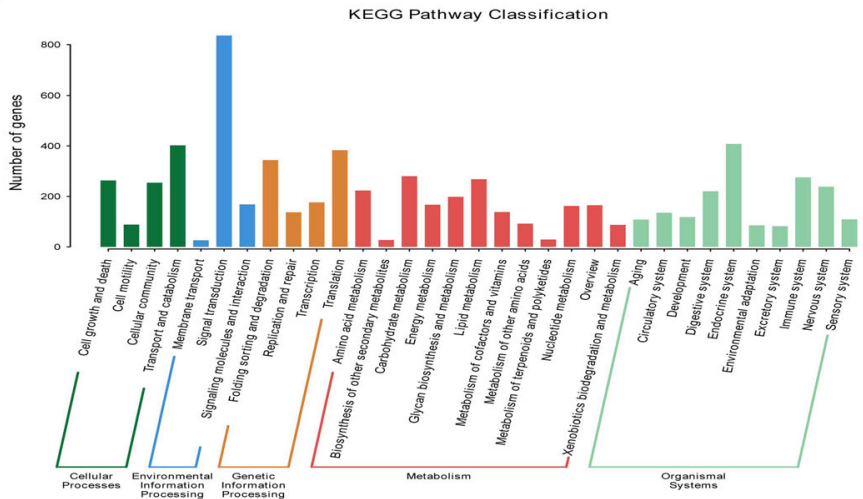

Fig. 3 The overall functional annotation of the assembled unigenes. a: The distribution of matched species of the unigenes according to the NR database. $\mathbf{b}$ : The overall GO classification annotation of the unigenes. $\mathbf{c}$ : The overall KOG functional classification of the unigenes. $\mathbf{d}$ : The overall KEGG pathway classification of the unigenes 
polyphemus (1021 unigenes) (Supplementary Table S5 and Fig. 3a). As a species in the phylum Arthropoda, the sequences of $P$. clarkii were similar to known sequences of other species of Arthropoda and Hydrozoa. Among the blast species, only 256 unigenes from RNA-seq data were matched with known genes of $P$. clarkii from the NR database (Supplementary Table S5). When the duplicate genes were removed, only 113 genes were matched (Supplementary Table S4), indicating that most of the genes expressed in the ovary of $P$. clarkii remain largely unknown.

In the GO functional classification analysis, a total of 19,773 unigenes were annotated to at least one GO term; all the GO annotated unigenes were classified into $68 \mathrm{GO}$ terms belonging to the three main categories of molecular function, biological process, and cellular components (Fig. 3b and Supplementary Table S6). Within the molecular function category, the binding $(13,063$ unigenes, 66.04\%) and catalytic activity (8656 unigenes, $43.78 \%)$ were the most dominant subcategories. The biological process category was the most frequently annotated, and the cellular process (11,953 unigenes, 60.45\%), metabolic process (9391 uningenes, 47.49\%), biological regulation (7393 unigenes, 37.39\%), and regulation of biological process (6816 unigenes, 34.47\%) were the most common subcategories. The cellular component was the second most frequently annotated category, and the cell $(12,162$ unigenes, $61.51 \%)$, cell part $(12,144$ unigenes, 61.42\%), organelle (9691 unigenes, $49.01 \%$ ), and membrane (6680 unigenes, $33.78 \%$ ) were the most common subcategories (Fig. 3b). In the biological process category, the subcategory of developmental process had 3930 (19.88\%) unigenes associated with ovarian development, and the subcategories of reproduction and reproductive process had 1002 (5.07\%) and 997 (5.04\%) unigenes, respectively, associated with reproduction (Fig. $3 \mathrm{~b})$. In order to understand the functions of lineal homologous genes of $P$. clarkii from other species, the KOG analysis was performed and the 10,520 annotated unigenes were divided into 25 functional categories. The results showed that the cluster of transcription (1854 unigenes, $17.62 \%$ ) was the most dominant in the ovary of $P$. clarkii, followed by signal transduction mechanisms (1817 unigenes, 17.27\%), general function prediction only (1437 unigenes, 13.66\%), and posttranslational modification, protein turnover, chaperones (984 unigenes, 9.35\%) (Fig. 3c and Supplementary Table S7). In addition, to understand the biological pathways of the unigenes, 3560 unigenes annotated in the KEGG database were analyzed and were assigned to 275 pathways, the number of unigenes in each pathway ranged from 1 to 135; the PI3-Akt signaling pathway (ko04151), endocytosis (ko04144), purine metabolism (ko00230) and RNA transport (ko03013) were the distinct pathways
(Supplementary Table S8). In the KEGG pathway, the cellular processes, environmental information processing, genetic information processing, metabolism and organismal systems were identified, and these comprised 4, $3,4,12$, and 10 subgroups, respectively, of which signal transduction, endocrine system, transport and catabolism, and translation were prominent (Fig. 3d and Supplementary Table S8).

\section{Correlation analysis of $P$. clarkii ovarian samples at different stages}

The 12 ovaries of $P$. clarkii were divided into four stages according to color, size, and histomorphology (Fig. 1). The stages were defined as stage I (I_7, I_19 and I_20), stage II (II_17, II_27 and II_30), stage III (III_33, III_49 and III_52), and stage IV (IV_35, IV_36 and IV_37). After the RNA-seq analysis, the repeatable correlation among all the samples was performed, and the Pearson, Kendall, and Spearman coefficient were calculated. The Pearson coefficients between different samples within one group of stages I-III were greater than 0.8 , representing extremely strong positive correlations; the Pearson coefficients between each sample of stage IV were greater than 0.6, showing strong positive correlations (Supplementary Figure S3). The Pearson coefficients demonstrated that stage IV was the most distant from stage I and stage II, and stage II was similar to stage III (Fig. 4a and Supplementary Figure S4A). IV_36 was different from IV_35 and IV_37 at stage IV (Fig. 4a and Supplementary Figure S4B). The Principal Component Analysis (PCA) showed that the samples from one stage could be distinguished from other stages (Fig. 4b and Supplementary Figure S4C). The algorithm of Bray Curtis was used to calculate the distance between different samples, and the Bray tree was constructed according to the distances. The results showed that stage I and stage II were in the same cluster, and stage III (III_33 and III_ 52) and stage IV were in another cluster, while III_49 (stage III) was in the stage II cluster (Fig. 4c). The Bray tree of the merged group also showed that stage IV was the most distant from other stages, and stage II and stage III were in the same cluster (Supplementary Figure S4D). In order to compare the distribution difference of distances within and among different groups, distance boxplots were drawn; these showed that the differences in distance between stages I, II and III were small, but there was one sample (IV_36) that was far from the other two samples in stage IV (Fig. 4d). To determine whether the grouping was meaningful, a non-parametric analysis of similarity (Anosim) was performed. If the $\mathrm{R}$ value is near 1 , the difference among groups is greater than that within groups; in contrast, when the $R$ value is near 0 , there is no significant difference within and among groups. The $\mathrm{R}$ value from the Anosim among the 


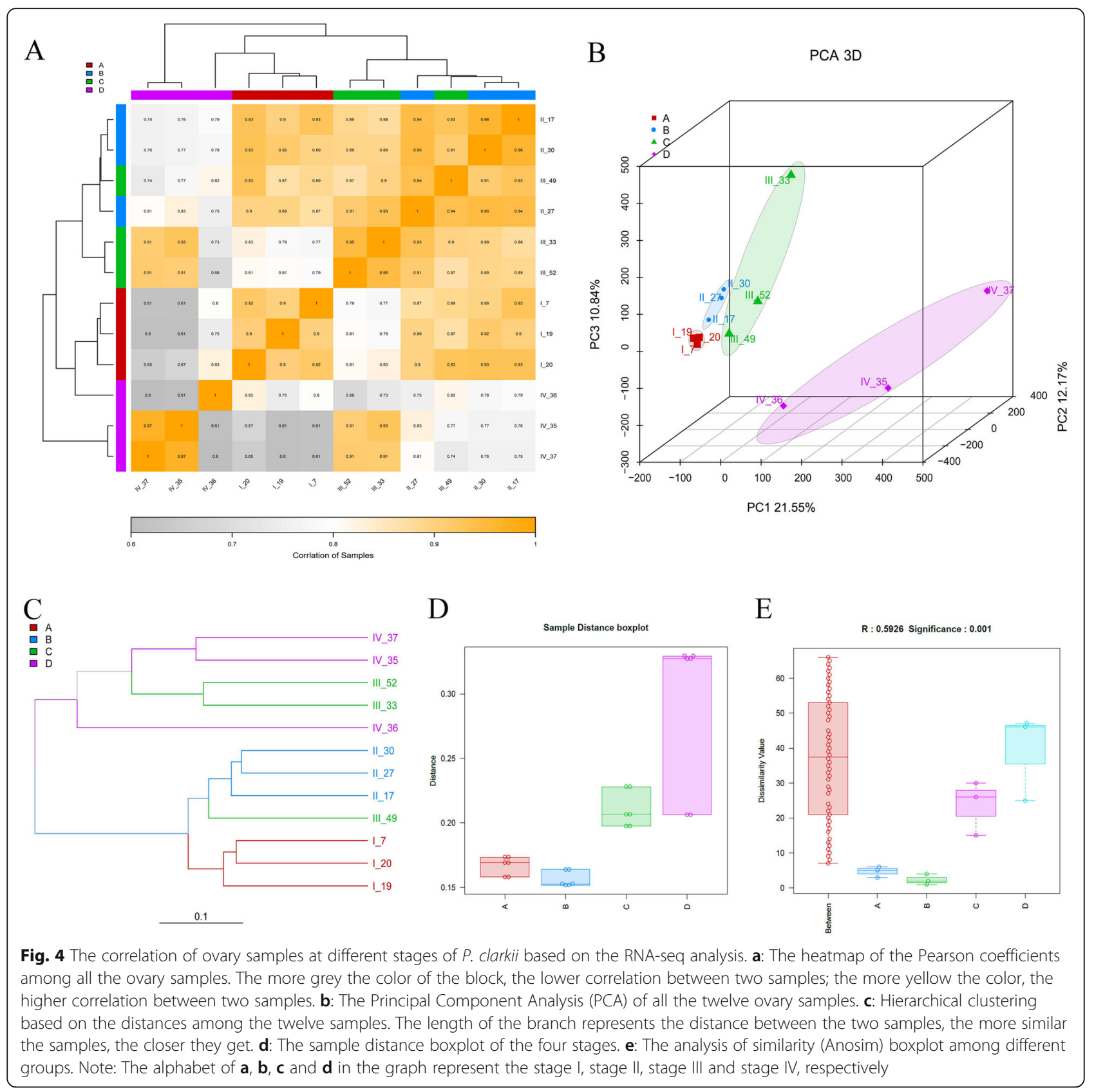

four groups was $0.5926(p<0.001)$ (Fig. 4e), indicating that the difference among groups was greater than that within groups. These results demonstrated that the grouping of four stages in this study was reliable, and the characteristics of the four stages of $P$. clarkii ovaries were different from each other.

\section{Differential expression analysis of $P$. clarkii ovarian samples at different stages}

The 216,444 unigenes were obtained from the twelve ovarian samples of P. clarkii, and the TPM values and annotations of the highly expressed unigenes are listed in Supplementary Table S9. The TPM values represent the expression levels of genes. The four stages were employed for pairwise comparisons using five comparison groups, including stage II_vs_stage I (B_vs_A), stage III_vs_stage I (C_vs_A), stage IV_vs_stage I (D_vs_A), stage III_vs_stage II (C_vs_B), and stage IV_vs_stage III (D_vs_C). The TPM density distribution revealed that $\log _{2}$ (TPM) values of the twelve ovarian samples mainly ranged from -5 to 5 , with only a small fraction in the range -15 to -12 . The $\log _{2}$ (TPM) values of IV_35, IV_ 

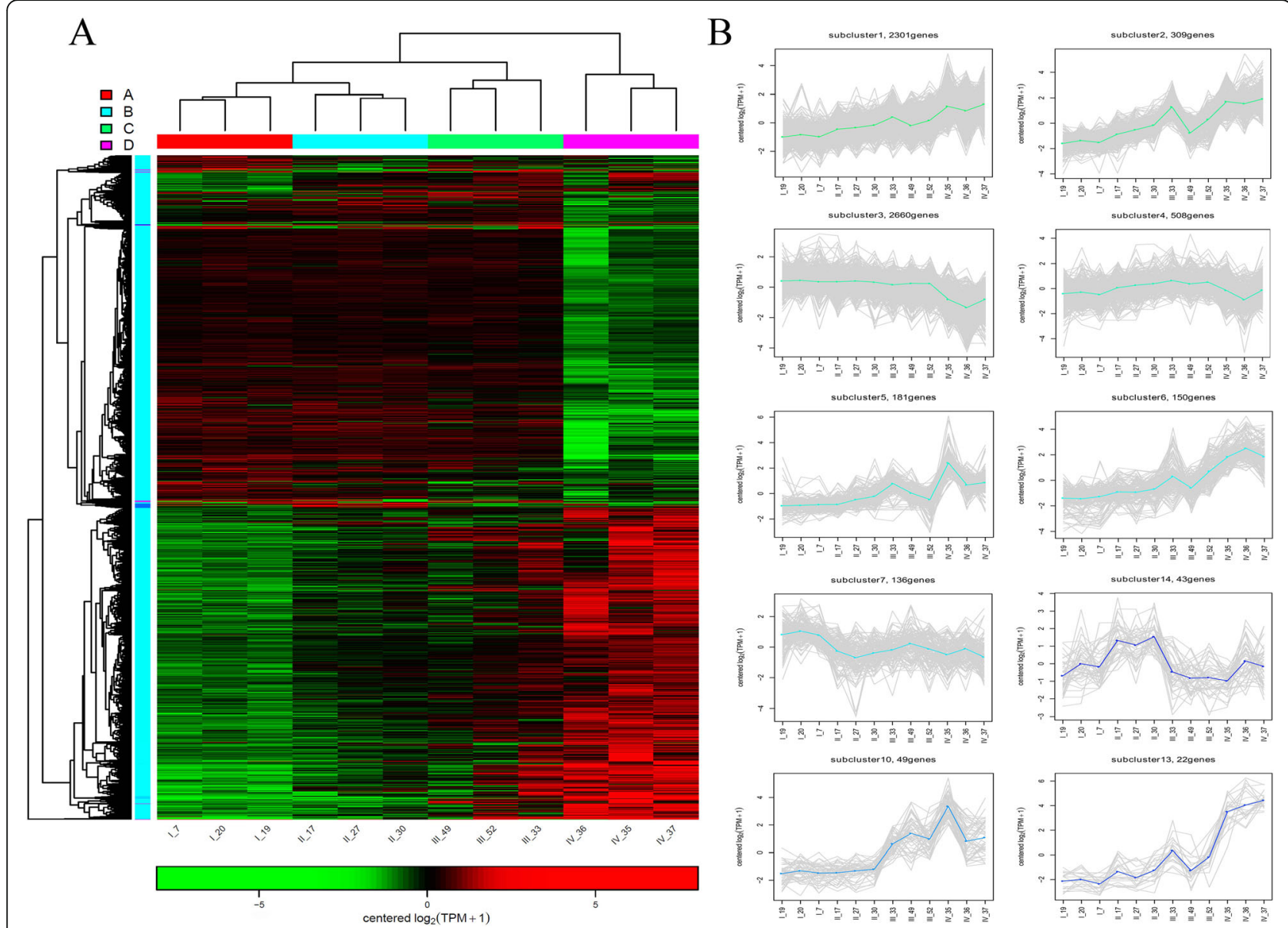

Fig. 5 The gene expression pattern of DEGs of twelve ovary samples of P. clarkii. a: The cluster heatmap of DEGs among the twelve ovarian samples. Groups A, B, C and D represent stage I, stage II, stage III and stage IV, respectively. The green color in the matrix represents downregulation of unigene, the red color in the matrix represents upregulation of unigene. $\mathbf{b}$ : The gene expression patterns within regular subclusters

36, and IV_37 of group D were the largest, indicating that the gene expression of stage IV was different from those of other stages (Supplementary Figure S5A). The TPM density distribution between groups demonstrated that the gene expression of one group was different from other groups, and the order of expression level was $\mathrm{D}>$ $\mathrm{C}>\mathrm{B}>\mathrm{A}$ (Supplementary Figure S5B-F).

Further, the cluster heatmap of DEGs based on the twelve ovarian samples was drawn (Fig. 5a). The results illustrated that the expression characteristics of IV_35, IV_36, and IV_37 in group D were clearly distinguished from the other samples. The expression characteristics of II_17, II_27, and II_30 in group B were similar with III_33, III_49, and III_52 in group C. According to the expression pattern in the cluster heatmap, all the DEGs were categorized into 30 subclusters (Fig. 5a), including 10 subclusters with regular trends (Fig. 5b) and 20 subclusters with irregular trends (Supplementary Figure S6). Among the regular subclusters, the expression trend of subcluster 1 (2301 genes) continuously increased from stage I to stage IV; the subcluster 3 (2660 genes) showed that the gene expression from stage I to stage III presented as a horizontal line, while being sharply decreased at stage IV; the genes in subcluster 7 (136 genes), subcluster 14 (43 genes), subcluster 10 (49 genes), and subcluster 13 (22 genes) were uniquely highly expressed in stage I, stage II, stages III-IV, and stage IV, respectively (Fig. 5b), illustrating that these genes had stage specificity. In addition, the number of DEGs between different groups revealed that the number of DEGs in D_vs_A was the highest, followed by D_vs_C and C_vs_ A. The numbers of DEGs in C_vs_B and B_vs_A were relatively low (Fig. 6a and Supplementary Table S10). The volcano plot listed the number of DEGs in five comparison groups, the comparisons of B_vs_A, C_vs A, D_vs_A, C_vs_B, and D_vs_C had 234, 694, 3046, 208 , and 674 significantly up-regulated genes, respectively, and 129, 196, 2706, 110, and 1818 significantly 


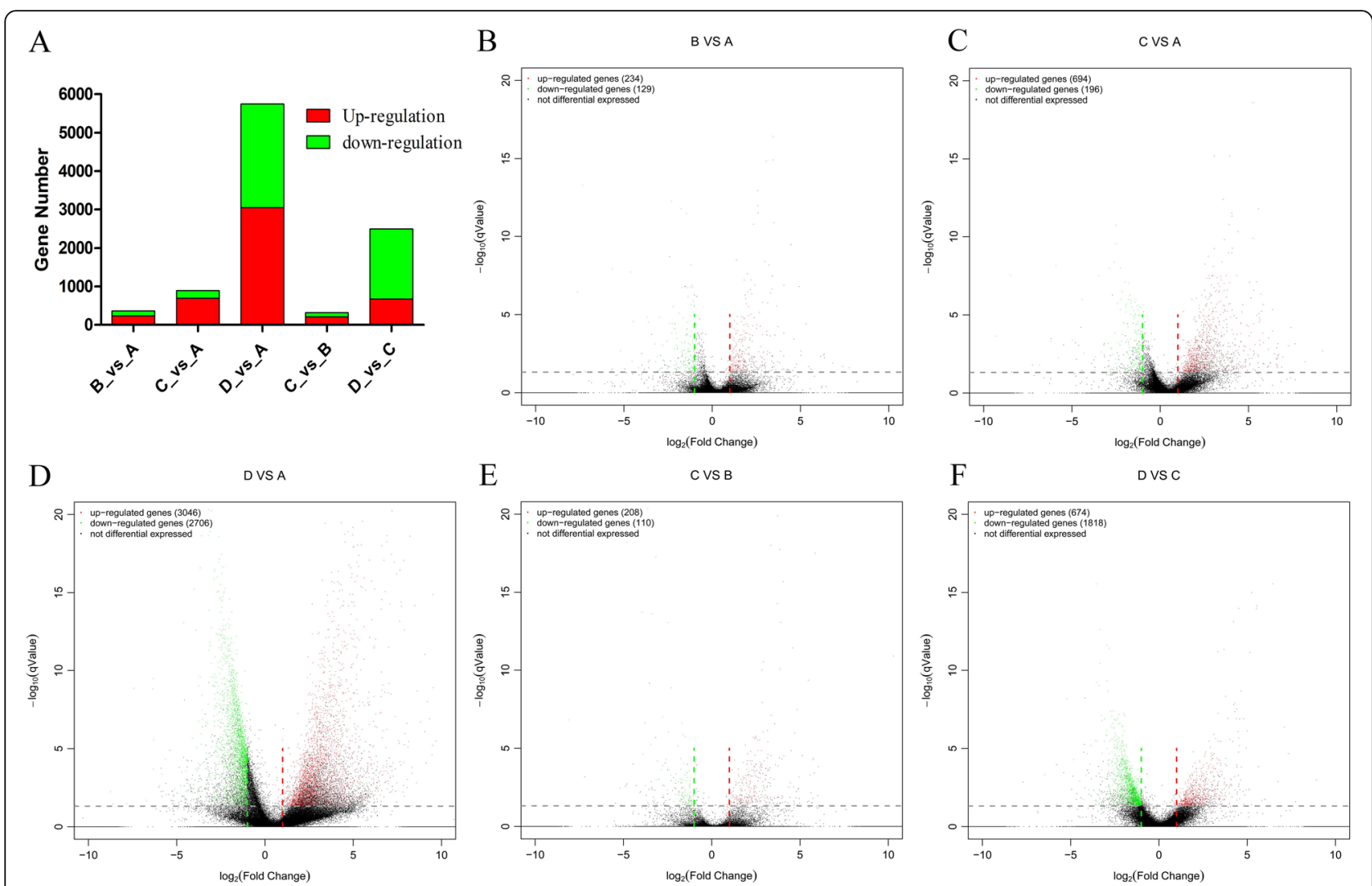

Fig. 6 The visualization analysis of expression differences among different comparisons. $\mathbf{a}$ : The barplot of the DEGs in five comparisons. B_vs_A is stage II_vs_stage I, C_vs_A is stage III_vs_stage I, D_vs_A is stage IV_vs_stage I, C_vs_B is stage III_vs_stage II, D_vs_C is stage IV_vs_stage III. b: The DEGs volcano of stage II_vs_stage I. c: The DEGs volcano of stage III_vs_stage I. d: The DEGs volcano of stage IV_vs_stage I. e: The DEGs volcano of stage III_vs_stage II. $\mathbf{f}$. The DEGs volcano of stage IV_vs_stage III. One dot in the volcano represents one gene, red dots represent upregulated genes and green dots represent down-regulated genes, black dots indicate the undifferentiated genes. The smaller the qValue, the larger the -log (qValue), the more significant the difference of the DEGs

down-regulated genes, respectively (Fig. 6b-f). Simultaneously, the DEGs heatmap of five comparison groups also confirmed that the difference between group D and group A was the most conspicuous, followed by between group D and group C (Fig. 7a). According to the information of DEGs, the Venn diagram was conducted, which could observe the DEGs distribution among different comparison groups, in the up-regulated DEGs, there were five core genes up-regulated in all five comparison groups (Fig. 7b), but no core gene was downregulated in any of the five comparison groups (Fig. 7c). These data demonstrated that the expression patterns among stages I, II, III, and IV of P. clarkii ovaries were different, and the number of DEGs continuously increased with the development of the ovary.

\section{Functional annotation of differentially expressed genes}

After selecting the DEGs, the distributions and enrichments of DEGs in the GO and KEGG functional categories were performed to discover the gene sets with differential expression levels. The enrichment analysis could identify the biological pathways that were most relevant to the biological phenomena, while the subcategory is thought to be significant enrichment if the Q value is less than 0.05 . The GO classification of DEGs showed the distribution among three main categories (biological process, cellular component, and molecular function), the results indicated that the numbers of DEGs in most of the subcategories for the B_vs_A and C_vs_B comparison groups were relatively low; the numbers of DEGs in the C_vs_A and D_vs_C comparison groups were considerably increased, and the DEGs number in D_vs_A comparison group was obviously greater than in other groups (Supplementary Figure S7). The DEGs of biological process in all comparison groups were mainly distributed in the terms of cellular process, metabolic process, and biological regulation; the DEGs of cellular component in all comparison groups mainly reflected on the terms of cell, organelle and membrane; the DEGs of molecular function in all comparison 


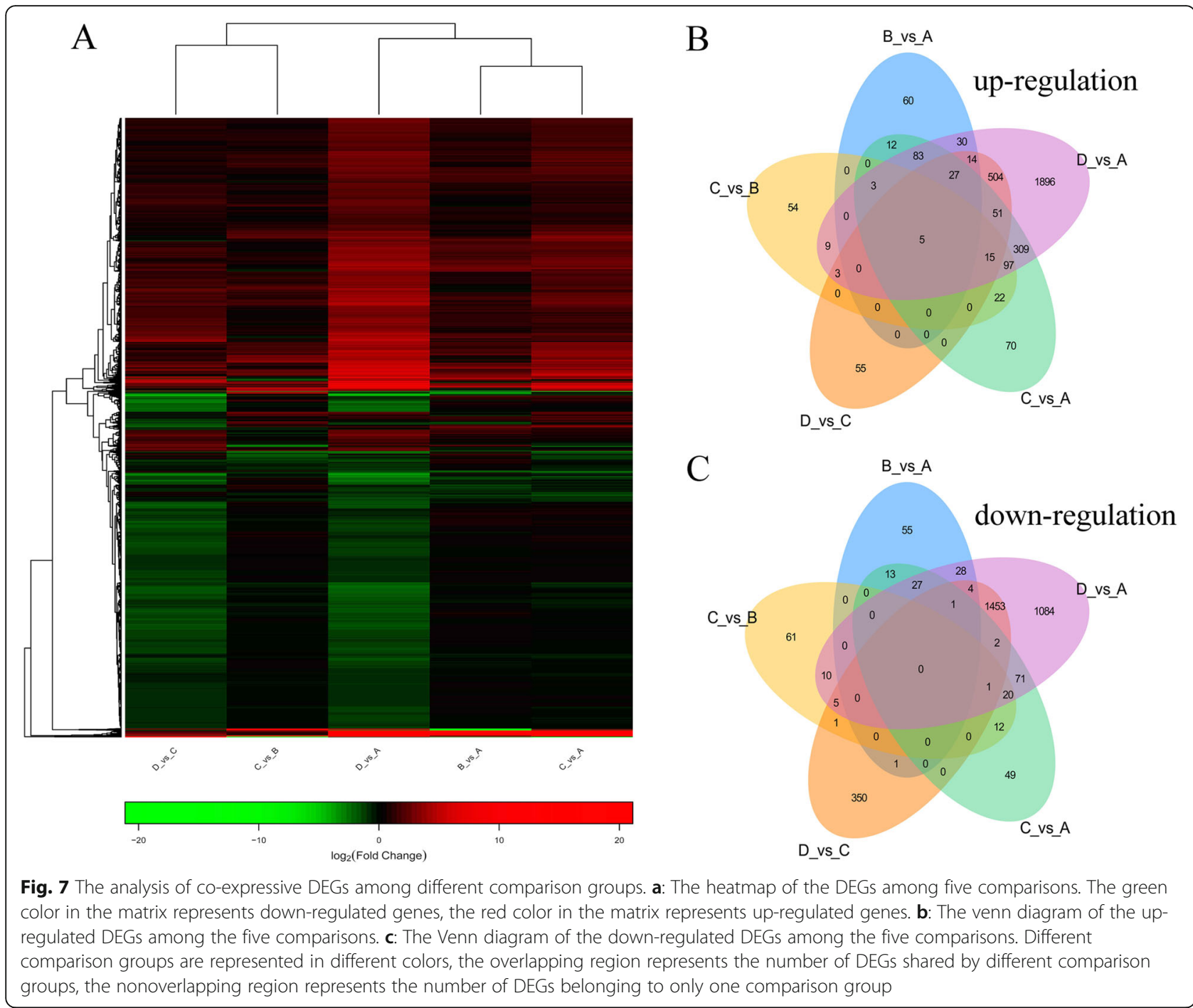

groups mainly located in the terms of binding, catalytic activity, and transporter activity (Supplementary Figure S7 and Supplementary Table S11). Furthermore, the DEGs of most of the terms in B_vs_A, C_vs_A and C_ vs_B comparisons were dominated by up-regulation, while the DEGs in D_vs_A and D_vs_C comparisons were dominated by down-regulation (Supplementary Table S11), illustrating that the some GO terms increased from stage I to stage III, but decreased at stage IV. Interestingly, the nutrient reservoir activity of molecular function in B_vs_A and C_vs_A comparisons had six up-regulated DEGs and was significant $(\mathrm{Q}$ value < 0.05) in the functional enrichment, but had no DEG in other comparisons (Supplementary Table S11), implying that the nutrient reservoir activity at stage II played a foreshadowing role for subsequent ovarian development. Within the biological process category, 1002 unigenes associated with reproduction (GO:0000003) were annotated, and the B_vs_A, C_vs_A, D_vs_A, C_vs_B, and D_
vs_C comparisons had $13,30,153,12$, and 81 significant unigenes, respectively, but the $\mathrm{Q}$ values in all comparisons were greater than 0.05 (Supplementary Table S11 and S12). The GO enrichment analysis demonstrated that the B_vs_A comparison had some significantly enriched GO terms, but the number was less than that of the C_vs_A comparison, while the C_vs_B comparison had only one significantly enriched GO term (extracellular region, GO:0005576). The GO enrichment of D_ vs_A was similar to D_vs_C (Fig. 8 and Supplementary Table S12). The B_vs_A, C_vs_A, D_vs_A, C_vs_B, and D_vs_C comparisons possessed 15, 29, 128, 1, and 133 significant GO terms, respectively (Supplementary Table S12). These data demonstrated that the difference between stage IV and stage III was the most obvious, and cell (GO:0005623), intracellular (GO:0005622), and organelle (GO:0043226) were the prominent GO terms, implying that the unigenes in these GO terms participated in the ovary maturation at stage IV. 


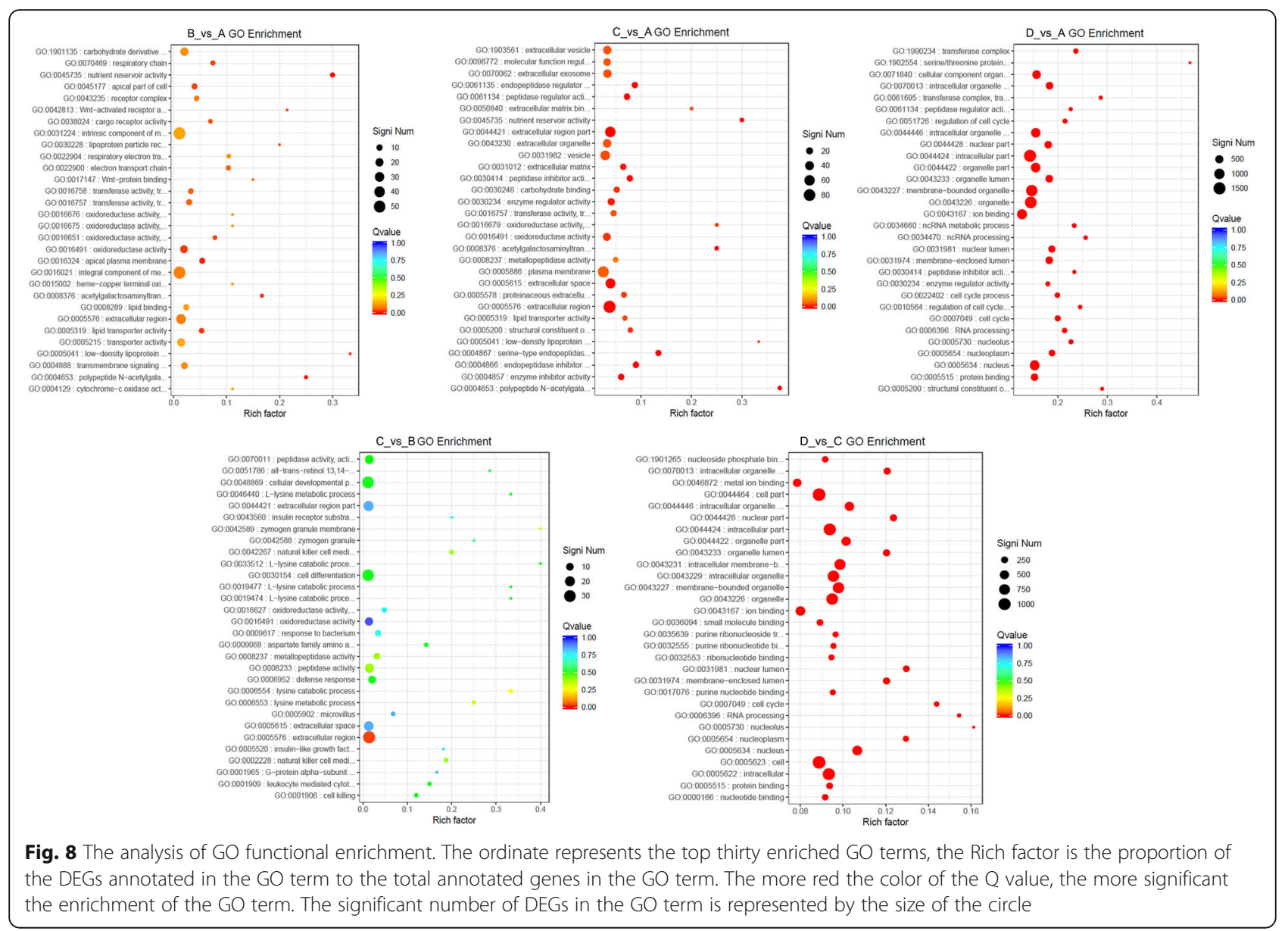

In addition, the KEGG classification of DEGs displayed the distribution of five main categories (cellular processes, environmental information processing, genetic information processing, metabolism, and organismal systems) (Supplementary Figure S8 and Supplementary Table S13). The subgroup of signal transduction belonging to environmental information processing was relatively prominent in all five comparisons; the significant numbers of each subgroup in D_vs_A and D_vs_C were greater than in the other three comparison groups, as in the analysis for the corresponding GO categories. However, the KEGG enrichment analysis disclosed that seven significantly enriched subgroups (apoptosis, ko04210; lysine biosynthesis, ko00300; NF-kappa B signaling pathway, ko04064; antigen processing and presentation, ko04612; TNF signaling pathway, ko04668; lysine degradation, ko00310; and renin secretion, ko04924) were observed in C_vs_B comparison group, more than in the other four comparison groups, indicating that these subgroups were responsible for the transition from stage II to stage III. The B_vs_A, C_vs_A, D_vs_A, and D_vs_C comparison groups had only two (PI3K-Akt signaling pathway, ko04151; focal adhesion, ko04510), one (lysosome, ko04142), two (basal transcription factors, ko03022; cell cycle, ko04110), and two (cell cycle, ko04110; p53 signaling pathway, ko04115) significantly enriched subgroups, respectively (Fig. 9 and Supplementary Table S14). These results revealed that the early development of $P$. clarkii ovary was mainly associated with the PI3K-Akt signaling pathway and focal adhesion, the middle development of the ovary was related to apoptosis, lysine biosynthesis, and NF-kappa B signaling pathway, and the late development of the ovary was involved with cell cycle and p53 signaling pathway.

\section{RT-qPCR confirmation of RNA-seq data}

In order to verify the accuracy of the RNA-seq data in this study, RT-qPCR was conducted to determine the expression levels of the nine genes C-type lectin (Lectin C, TRINITY_DN50699_c3_g1), Kazal type serine protease inhibitors (KSPI, TRINITY_DN50830_c1_g3), Astacin (TRINITY_DN42990_c1_g1), Prostaglandin G/H synthase 2 (PGHS2, TRINITY_DN42030_c3_g1), Vitellogenin (TRINITY_DN52153_c0_g3), Serine proteinase inhibitors (SERPIN, TRINITY_DN43168_c6_g1), Venom allergen 5 (VA5, TRINITY_DN38550_c1_g2), 


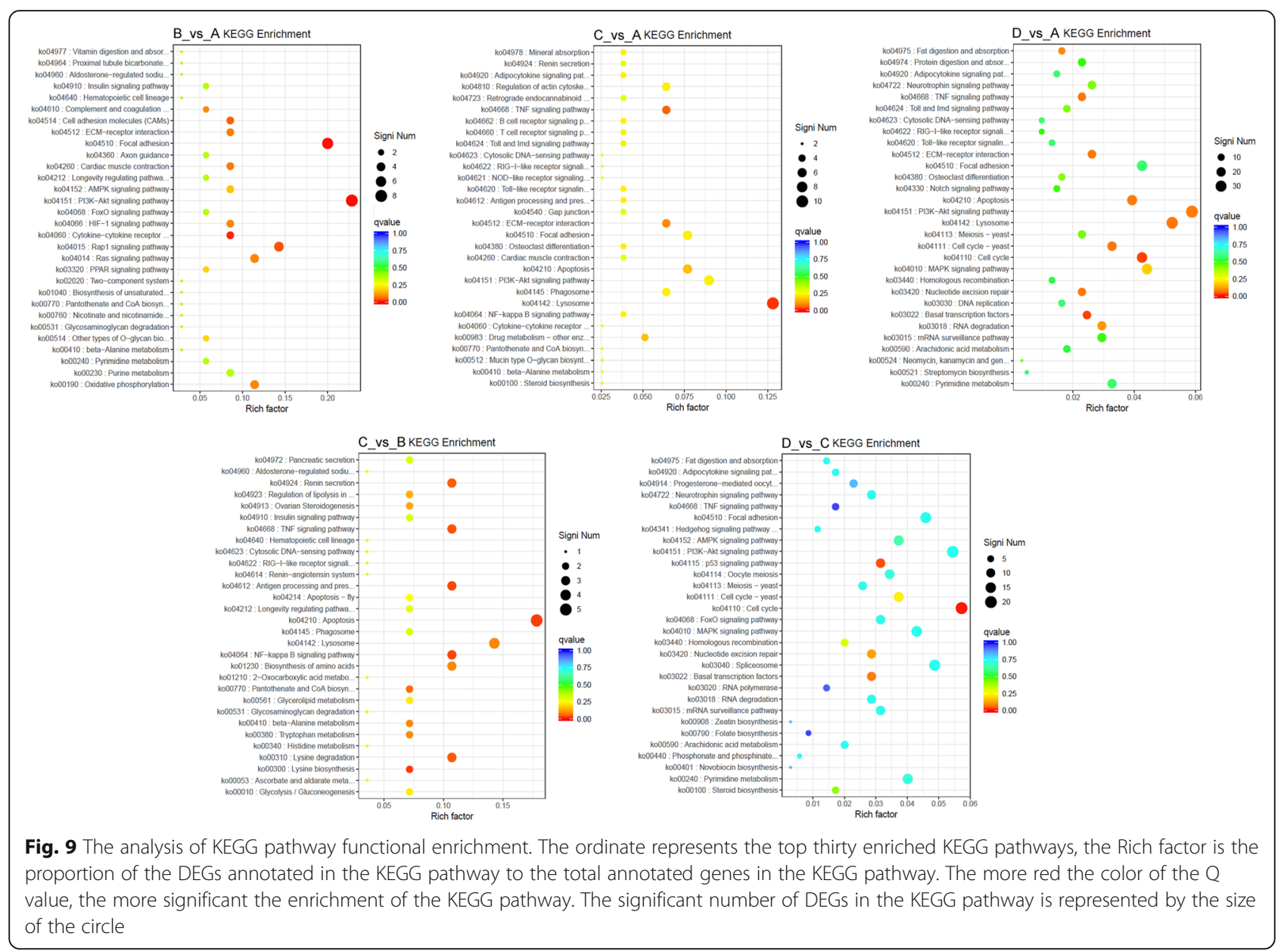

Cysteine-rich secretory proteins (CRISP, TRINITY DN43203_c3_g2), and hyperglycemic peptide 2 precursor (HGP2P, TRINITY_DN49383_c1_g2), using the ovaries from stage I to stage IV of $P$. clarkii. Three DEGs (VA5, CRISP, and HGP2P) that were up-regulated in all five comparisons, were purposefully selected; the remaining six DEGs (Lectin C, KSPI, Astacin, PGHS2, Vitellogenin, and SERPIN) were randomly selected from the DEGs dataset, three genes were down-regulated and three genes were up-regulated. GAPDH (TRINITY DN47812_c1_g3) was used as internal reference gene for normalization, because GAPDH was stably expressed in ovary tissues of $P$. clarkii as shown in the RNA-seq data (Supplementary Table S14). The results showed that the change trends of these nine genes detected by RT-qPCR were consistent with those from the RNA-seq data in all five comparison groups (Fig. 10), confirming that the RNA-seq data were authentic.

\section{Discussion}

The red swamp crayfish Procambarus clarkii is one of the most well-known invasive species, and it has become an economically important aquatic resource [9]. The delicious taste and high nutritive value are the major reasons for gaining in popularity in China. At present, increasing numbers of farmer are raising P. clarkii, and the crayfish-rice culture is the main farming pattern [17]. The production level is continuously increasing, thereby improving the income of farmers. The ovarian development of female $P$. clarkii is an important physiological process representing the reproductive capacity. However, the integrated reproductive pattern of $P$. clarkii remains largely unknown, particularly the molecular mechanisms of ovarian development. This lack of knowledge hinders the production of $P$. clarkii to a large extent. In order to obtain information concerning the molecular mechanisms of ovarian development, we captured the transcriptomics data of four ovarian developmental stages of $P$. clarkii using NGS and compared the difference between different stages in order to understand the developmental pattern.

The ovary of $P$. clarkii consists of oocytes and follicle cells surrounded by a membrane composed of connective tissue and epithelium. The previous studies reported 


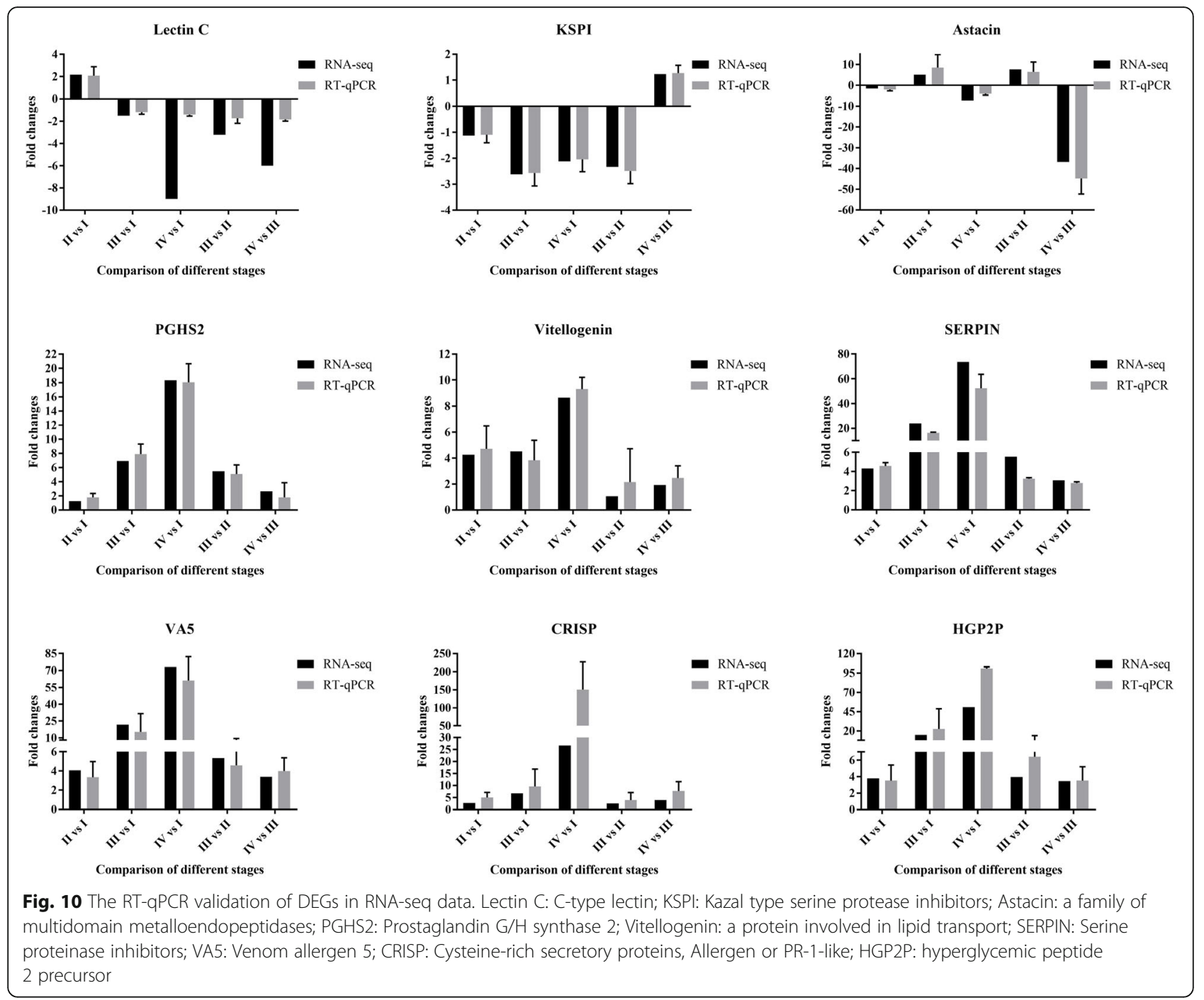

that the ovarian maturation of crayfish proceeds in several stages that can be identified based on morphology and coloration, exhibiting an increase in the size of the ovary as the oocytes proliferate and display uptake of yolk and lipovitellin. The ovarian color changes from light to dark in the order of transparent, translucent white, opaque ivory, yellow, and dark brown [42, 43]. In this study, except for the non-developed ovaries that were transparent, we observed the same color changes of ovaries, and the ovary size continuously increased during the change from white to brown status. We classified the pattern into four stages, the previtellogenic stage (stage I, white), the early vitellogenic stage (stage II, yellow), the middle vitellogenic stage (stage III, light brown), and the mature stage (stage IV, dark brown). Kulkarni et al. (1991) classified the oocyte development of $P$. clarkii into seven stages, and the size of the oocyte increased along with development, including oogonial
$(<10 \mu \mathrm{m})$, immature $(10-65 \mu \mathrm{m})$, avitellogenic (66$160 \mu \mathrm{m})$, early vitellogenic $(161-245 \mu \mathrm{m})$, midvitellogenic $(246-455 \mu \mathrm{m})$, late vitellogenic $(456-980 \mu \mathrm{m})$, and postvitellogenic-resorptive stages [41]. In the present study, we also found that the size of oocytes of $P$. clarkii increased along with the ovarian development from stage I to stage IV. The sizes of oocytes at stage I in this study was $100-200 \mu \mathrm{m}$, corresponding to the avitellogenic stage; however, the sizes at later stages were larger than those at the corresponding stages reported by Kulkarni et al. (1991) [41]. The sizes of middle vitellogenic stage III and mature stage IV in our study were 500$1000 \mu \mathrm{m}$ and $>1000 \mu \mathrm{m}$, respectively. The reasons for the discrepancy might be related to the samples being collected from different areas and at different times. Moreover, the sizes of most oocytes in the same ovary were generally uniform, but some smaller or immature oocytes could also be found in a nearly mature ovary 
[41]. We also found some immature oocytes in mature ovaries as in previous results, indicating that the oocyte development was asynchronous.

In this study, we performed the transcriptomics of twelve ovaries at four stages, and the total clean read counts of the twelve samples ranged from 42,013,648 to $62,220,956$. The total number of bases ranged from 6, $118,532,265$ bp to $9,054,802,655 \mathrm{bp}$. The Q30 percentage (94.52-95.58\%) of our result was slightly higher than the previous result $(91.69 \%)$ for mature ovaries of $P$. clarkii [49], demonstrating that our sequencing data were more accurate. The average GC base ratio was $46.99 \%$ in our study, which was lower than the result reported by Kang et al. (2019) [49]. The number of samples in our experiment and the Q30 percentage were greater than in the previous assays, and thus we speculated that the GC ratio of our result was more reliable. Jiang et al. (2014) used 454 pyrosequencing to obtain 10,748 isotigs with an N50 of $1794 \mathrm{bp}$ in the ovary library [39]; simultaneously, Shen et al. (2014) used Illumina HiSeq 2000 instrumentation to capture an increasing number of 50 , 219 non-redundant genes [37]. However, five years later, Kang et al. (2019) used Illumina HiSeq 4000 instrumentation to acquire a total of 105, 957 transcripts $(>200$ bp) with an N50 of $1862 \mathrm{bp}$ and 69,261 unigenes with an N50 of 1129 bp [49], far exceeding the results reported by Jiang et al. (2014) [39] and Shen et al. (2014) [37]. In this study, we employed an Illumina HiSeq 2500 to obtain 445,326 transcripts and 216,444 unigenes, of which, 26,934 unigenes comprised more than $1000 \mathrm{bp}$, more than the previous results mentioned above. Although the N50 values of transcripts and unigenes were $1858 \mathrm{bp}$ and $912 \mathrm{bp}$, respectively, most of the unigenes (87.56\%) were distributed between $200 \mathrm{bp}$ and $1000 \mathrm{bp}$, similar to the result of Kang et al. (2019) [49]. These data demonstrate that the sequencing information from the Illumina HiSeq 2500 was of high quality and had more content for analysis of the characteristics of the P. clarkii ovary. In the transcriptomic research between testis and ovary of P. clarkii, 15,667 (69.16\%), 13,818 (61.00\%), 12,419 (54.83\%), 7243 (31.98\%), and 6902 (30.47\%) genes within the 22,652 annotated genes could be matched with known genes in the NR, Swiss-Prot, KEGG, COG, and GO databases, respectively [39]. Recently, the transcriptomic data of the gills of $P$. clarkii produced 13,948 (40.5\%), 5201 (15.1\%), 10,747 (31.2\%), 13,084 (37.99\%), 6381(18.52\%), and 13,084 (37.99\%) unigenes annotated in NR, NT, Swiss-Prot, PFAM, KOG, and GO databases, respectively [32]. In our transcriptomic data, there were similar numbers of annotated genes in all databases, but due to the high number of unigenes in our results, the annotated proportion was lower than in other reports. However, the number of unigenes annotated in at least one database was higher than in the other reports mentioned above. The best matched species in our data were Zootermopsis nevadensis, Hydra vulgaris, Limulus polyphemus, and Daphnia magna, different from the Daphnia pulex, Tribolium castaneum, Pediculus humanus corporis, and Nasonia vitripennis reported by Jiang et al. (2014) [39] and Daphnia pulex, Tribolium castaneum, and Pediculus humanus reported by Shen et al. (2014) [37]. The main similarity was that most of these species belong to the Arthropoda.

The correlation analysis of the twelve ovary samples based on the RNA-seq data was analyzed in our study, the three samples in the same stage had high Pearson coefficients $(>0.6)$, demonstrating that the different samples in the same group were highly correlated. The Pearson coefficients indicated that stage IV was far removed from other stages, and stage II was similar to stage III. In addition, the PCA also showed that each stage could be distinguished from the other stages, and the $\mathrm{R}$ value of the Anosim was 0.5926 and highly significant $(p<$ 0.001 ). These data demonstrated that the grouping of the four developmental stages in this study was reliable. The DEG analysis could help to identify the differences in gene expressions in different samples, thereby confirming the relationship between genotype and phenotype. Shui et al. (2012) identified 13 down-regulated and 9 up-regulated proteins of the vitellogenic ovary compared with the previtellogenic ovary of $P$. clarkii through two-dimensional gel electrophoresis, and this result could be helpful for understanding the proteins involved in ovarian development [48]; however, the number of DEGs identified was small. In this study, we found a greater number of DEGs between early vitellogenic ovaries (stage II) and previtellogenic ovaries (stage I) by NGS technology, demonstrating that the results from this study could provide more information for analyzing ovarian development. Furthermore, among the four different stages, we found that the difference between the mature ovary (stage IV) and the previtellogenic ovary (stage I) was greater than in other comparisons, the second largest difference was between the mature ovary (stage IV) and the middle vitellogenic ovary (stage III), while the difference between the middle vitellogenic ovary (stage III) and the early vitellogenic ovary (stage II) was less than in other comparison groups. These findings demonstrated that the mature stage was the most important for ovulation through major changes involving many genes.

GO classification can be utilized to analyze the functional categorization of unigenes or DEGs in transcriptomic analysis of most species, including crustaceans. For Portunus trituberculatus, the molecular function (the binding and catalytic activity constituted the majority of the categories) was the most assigned, followed by biological process (the cellular process and metabolic 
process were the predominant terms) and cellular components (the cell, cell part, and organelle were the major categories in cellular components) [68]. In black tiger shrimp, the biological process was the highest annotated, followed by cellular component and molecular function, this was different from the Portunus trituberculatus results, while the subgroups in these three categories were the same as for Portunus trituberculatus [69]. Also, as a crustacean, the previous ovarian transcriptomic analysis of $P$. clarkii $[37,39,49]$ revealed that the GO annotation was similar to those of Portunus trituberculatus and the black tiger shrimp; as expected, we also got the similar results in the GO annotation in this study. The DEGs between different stages indicated that the differences among stages I, II and III were relatively low, while the difference between stages IV and III was more obvious than in other comparison groups of two adjacent stages. Notably, the GO term of nutrient reservoir activity within molecular function was significant at stages II and III compared with stage I, indicating that these stages provide enough nutrient for subsequent ovarian development. In addition, the GO terms of cell (GO: 0005623), intracellular (GO:0005622), and organelle (GO:0043226) were significantly prominent between stages IV and III, speculating that these GO terms played important roles in ovarian mature. Furthermore, to understand the complex biological processes of genes, the KEGG database was used to analyze functional information of metabolic pathways or regulatory networks of genes in cells. In this study, 3560 unigenes were annotated in the KEGG database; the subgroups of signal transduction, endocrine system, transport and catabolism, and translation were the most prominent, similar to the result of Meng et al. (2015) [68]. We found that the PI3-Akt signaling pathway (ko04151), endocytosis (ko04144), purine metabolism (ko00230), and RNA transport (ko03013) were the distinct pathways. Simultaneously, the PI3K-Akt signaling pathway, the Wnt signaling pathway, the GnRH signaling pathway, progesterone-mediated oocyte maturation, the insulin signaling pathway and the TGF-beta signaling pathway were identified, and these were related to gonadal development and maturation and were assigned to the subgroups of signal transduction and endocrine system as in previous studies $[68,69]$. In mammals, the PI3K-Akt signaling pathway is indispensable for the regulation of cell proliferation, survival, migration, and metabolism in different tissues, and it plays an essential role in oocyte growth and the activation of primordial follicles in the ovary [70-73]. Interestingly, we found that the PI3K-Akt signaling pathway was only significant at stage II compared with stage I, indicating that this pathway played a vital role for the activation of primordial follicles and oocyte growth in early vitellogenic ovaries of $P$. clarkii.
Furthermore, various "nutrient sensing" mechanisms might take part in forming the link between nutrient status and folliculogenesis, while the PI3K-Akt pathway could sense nutrient flux from within the follicle [74]. Amazingly, the GO term of nutrient reservoir activity was significant at stage II compared with stage I, corresponding to the PI3K-Akt pathway. Therefore, we speculated that the PI3K-Akt pathway could sense the nutrient reservoir activity to regulate oocyte growth and activation of primordial follicles in early vitellogenic ovaries of $P$. clarkii. Finally, apoptosis, lysine biosynthesis, and the NF-kappa B signaling pathway were significant at stage III compared with stage II, indicating that stage III was related to immune response and biosynthesis; cell cycle and the p53 signaling pathway were prominent at stage IV compared with stage III, showing that stage IV was involved with cell proliferation and DNA replication, corresponding to the GO term of cell cycle regulation reported by Jiang et al. (2014) [39]. These data demonstrated that different stages have different pathways for regulating the ovarian development of P. clarkii.

\section{Conclusion}

In summary, we compared the transcriptomics among four ovarian stages of $P$. clarkii through Illumina sequencing technology, including the previtellogenic stage, the early vitellogenic stage, the middle vitellogenic stage, and the mature stage. We found that the expression patterns of the first three stages defined in this study were similar, but each stage also had its own uniquely expressed modules that distinguished it from other stages. The expression pattern of the mature stage was quite different from those of the other three stages. The GO term of nutrient reservoir activity played a foreshadowing role at the early vitellogenic stage, and the terms of cell, intracellular, and organelle were prominent at the mature stage. The KEGG analysis revealed that the early ovarian development of $P$. clarkii was mainly associated with the PI3K-Akt signaling pathway and focal adhesion, while the middle ovarian development was related to apoptosis, lysine biosynthesis, and the NF-kappa $\mathrm{B}$ signaling pathway, and the late ovarian development was involved with the cell cycle and p53 signaling pathway. These data provide insights into the molecular mechanisms of ovarian development, and may help to improve the reproductive performance of $P$. clarkii.

\section{Supplementary Information}

The online version contains supplementary material available at https://doi. org/10.1186/s12864-021-07537-x.

Additional file 1: Supplementary Figure S1: The base content of twelve ovary samples. Supplementary Figure S2: The information of 
the assembly transcripts of the $P$. clarkii ovary. Supplementary Figure S3: The repeatable correlation of ovary samples at the same stage of $P$. clarkii. Supplementary Figure S4: The correlation of the different stages of $P$. clarkii ovary. Supplementary Figure S5: The TPM analysis of the different stages of $P$. clarkii ovary. Supplementary Figure S6: The gene expression patterns within irregular subclusters of DEGs of twelve ovary samples. Supplementary Figure S7: The GO classification of DEGs in different comparison groups. Supplementary Figure S8: The KEGG classification of DEGs in different comparison groups. Supplementary Table S1: Primers used for RT-qPCR.

Additional file 2.

Additional file 3.

Additional file 4.

Additional file 5.

Additional file 6.

Additional file 7.

Additional file 8.

Additional file 9.

Additional file 10.

Additional file 11.

Additional file 12.

Additional file 13.

Additional file 14 .

\section{Acknowledgements}

We would like to thank the staffs of the red swamp crayfish farm at Laibin Guangxi, China for catching crayfishes and providing accommodation and catering.

\section{Authors' contributions}

Yizhi Zhong and Zhuanling Lu designed the project, totally analyzed the transcriptomic data, performed the RT-qPCR for validation of RNA-seq data, and wrote the main manuscript text. Wenbin Zhao, Zhifa Lu and Yanling Yu collected the ovarian samples of $P$. clarkii. Wenbin Zhao, Zhangsheng Tang and Liming Huang performed the H\&E staining experiment and identified the four developmental stages of ovary. Xiangxing Zhu and Aifen Yan analyzed the information of assembly and the overall functional annotation based on the transcriptome data, and prepared Figs. 2, 3 and 4. Xiang Liang analyzed the DEGs and the gene expression pattern of different stages and prepared Figs. 5, 6 and 7. Dongsheng Tang performed GO enrichment and KEGG enrichment, and prepared Figs. 8 and 9. Dapeng Wang and Zhuanling Lu supervised the manuscript. All authors reviewed and approved the manuscript.

\section{Funding}

This work was supported by the Scientific and Technological Innovation and Industrialization Demonstration of "Double Hundred" Rice-Shrimp Ecological Breeding in Guangxi (2020AA13004AA), and supported by Programs of Guangxi Innovation and Promotion (AA17204095-4 and AA17204094-6). This work was jointly supported by the National Science and Technology Major Project (2009ZX08010-023B, 2014ZX08010-12B), the National Natural Science Foundation of China (82070199), the Guangdong Provincial R\&D Project in Key Areas (2018B020203003), and the Guangdong Basic and Applied Basic Research Fund (2019A1515110280).

\section{Availability of data and materials}

All data generated or analyzed during this study are available in this article and its supplementary information files. The transcriptomic raw data in the current study have been deposited to the NCBI SRA database with the accession number of PRJNA690634 (https://www.ncbi.nlm.nih.gov/sra/ PRJNA690634)

\section{Declarations}

Ethics approval and consent to participate Not applicable.
Consent for publication

Not applicable.

\section{Competing interests}

The authors declare that they have no competing interests.

\section{Author details}

${ }^{1}$ Guangxi Academy of Fishery Sciences/Guangxi Key Laboratory of Aquatic Genetic Breeding and Healthy Aquaculture, Nanning 530021, China. ${ }^{2}$ Guangdong Provincial Key Laboratory of Animal Molecular Design and Precise Breeding, Guangdong Provincial Engineering and Technology Research Center for Gene Editing, School of Medical Engineering, Foshan University, Foshan 528225, China. ${ }^{3}$ Development Research Institute of Agro-animal Husbandry Industry, Guangxi University, Nanning 530004, China.

Received: 30 December 2020 Accepted: 15 March 2021

Published online: 21 March 2021

\section{References}

1. Huner JV. Procambarus in North America and elsewhere. In: Holdich DM, Lowery RS, editors. Freshwater crayfish: biology, management and exploitation. Portland (OR): Timber Press; 1988. p. 239-61.

2. Elmossalami MK, Emara MT. Safety and quality of fresh water crayfish, Procambarus clarkii in the river Nile. Nahrung. 1999;43(2):126-8. https://doi. org/10.1002/(SICI)1521-3803(19990301)43:2<126::AID-FOOD126>3.0.CO;2-O.

3. Barbaresi S, Fani R, Gherardi F, Mengoni A, Souty-Grosset C. Genetic variability in European populations of an invasive American crayfish: preliminary results. Biol Invasions. 2003;5(3):269-74. https://doi.org/10.1 023/A 1026133519707

4. Cruz MJ, Rebelo R. Colonization of freshwater habitats by an introduced crayfish, Procambarus clarkii, in Southwest Iberian Peninsula. Hydrobiologia. 2007;575(1):191-201. https://doi.org/10.1007/s10750-006-0376-9.

5. Yi S, Li Y, Shi L, Zhang L, Li Q, Chen J. Characterization of population genetic structure of red swamp crayfish, Procambarus clarkii, in China. Sci Rep. 2018;8(1):5586. https://doi.org/10.1038/s41598-018-23986-z.

6. Yue GH, Li JL, Bai ZY, Wang CM, Feng F. Genetic diversity and population structure of the invasive alien red swamp crayfish. Biol Invasions. 2010;12(8): 2697-706. https://doi.org/10.1007/s10530-009-9675-1.

7. Souty-Grosset C, Anastácio PM, Aquiloni L, Banha F, Choquer J, Chucholl C, Tricarico E. The red swamp crayfish Procambarus clarkii in Europe: impacts on aquatic ecosystems and human wellbeing. Limnologica - Ecology and Management of Inland Waters. 2016;58:78-93. https://doi.org/10.1016/j. limno.2016.03.003

8. Bi K, Gu W, Wang W. Sensitive and rapid detection of freshwater crustacean Spiroplasmas by ISRs-sequence-targeted species-specific primers. Eur Food Res Technol. 2008;227(6):1733-7. https://doi.org/10.1007/s00217-008-0903-1.

9. Zhu BF, Huang Y, Dai YG, Bi CW, Hu CY. Genetic diversity among red swamp crayfish (Procambarus clarkii) populations in the middle and lower reaches of the Yangtze River based on AFLP markers. Genet Mol Res. 2013; 12(1):791-800. https://doi.org/10.4238/2013.March.13.8.

10. Gherardi F. Crayfish invading Europe: the case study of Procambarus clarkii. Mar Freshw Behav Physiol. 2006;39(3):175-91. https://doi.org/10.1080/1023 6240600869702

11. Li Y, Guo X, Cao X, Deng W, Luo W, Wang W. Population genetic structure and post-establishment dispersal patterns of the red swamp crayfish Procambarus clarkii in China. PLoS One. 2012;7(7):e40652. https://doi.org/1 0.1371/journal.pone.0040652.

12. Liu G, Zhou L. Population genetic structure and molecular diversity of the red swamp crayfish in China based on mtDNA COI gene sequences. Mitochondrial DNA A DNA Mapp Seq Anal. 2017;28(6):860-6. https://doi. org/10.1080/24701394.2016.1199022.

13. Zhong Y, Tang Z, Huang L, Wang D, Lu Z. Genetic diversity of Procambarus clarkii populations based on mitochondrial DNA and microsatellite markers in different areas of Guangxi, China. Mitochondrial DNA A DNA Mapp Seq Anal. 2020;31(2):48-56. https://doi.org/10.1080/24701394.2020.1721484.

14. Spielman D, Brook BW, Frankham R. Most species are not driven to extinction before genetic factors impact them. Proc Natl Acad Sci U S A. 2004;101(42):15261-4. https://doi.org/10.1073/pnas.0403809101.

15. Fisheries Department of the Chinese Ministry of Agriculture and Rural Affairs. The development report of red swamp crayfish industry (2019). 2019; http://www.yyj.moa.gov.cn/gzdt/201909/t20190910_6327557.htm. 
16. Jin S, Jacquin L, Xiong M, Li R, Lek S, Li W, Zhang T. Reproductive pattern and population dynamics of commercial red swamp crayfish (Procambarus clarkii) from China: implications for sustainable aquaculture management. PeerJ. 2019;7:e6214. https://doi.org/10.7717/peerj.6214.

17. Weimin M. Recent developments in Rice-fish culture in China: a holistic approach for livelihood improvement in rural areas. In: De Silva S.S., Davy F. B. (eds) Success stories in Asian aquaculture. Springer, Dordrecht. 2010; pp15-40, DOl: https://doi.org/10.1007/978-90-481-3087-0_2.

18. Huang J, Tang S, Cai F, Lin Y, Wu Z. Microsatellite evidence of dispersal mechanism of red swamp crayfish (Procambarus clarkii) in the Pearl River basin and implications for its management. Sci Rep. 2017;7(1):8272. https:// doi.org/10.1038/s41598-017-08552-3.

19. Ameyaw-Akumfi $C$, Hazlett BA. Sex recognition in the crayfish Procambarus clarkii. Science. 1975;190(4220):1225-6. https:/doi.org/10.1126/science.1198111.

20. Tattersall GJ, Luebbert JP, LePine OK, Ormerod KG, Mercier AJ. Therma games in crayfish depend on establishment of social hierarchies. J Exp Biol. 2012;215(Pt 11):1892-904. https://doi.org/10.1242/jeb.065946.

21. Buscaino G, Filiciotto F, Buffa G, Di Stefano V, Maccarrone V, Buscaino C, Mazzola S, Alonge G, D'Angelo S, Maccarrone V. The underwater acoustic activities of the red swamp crayfish Procambarus clarkii. J Acoust Soc Am. 2012;132(3):1792-8. https://doi.org/10.1121/1.4742744.

22. Leung TS, Naqvi SM, Naqvi NZ. Paraquat toxicity to Louisiana crayfish (Procambarus clarkii). Bull Environ Contam Toxicol. 1980;25(3):465-9. https:// doi.org/10.1007/BF01985555.

23. Barbee GC, McClain WR, Lanka SK, Stout MJ. Acute toxicity of chlorantraniliprole to non-target crayfish (Procambarus clarkii) associated with rice-crayfish cropping systems. Pest Manag Sci. 2010;66(9):996-1001. https://doi.org/10.1002/ps.1972

24. Al Kaddissi S, Frelon S, Elia AC, Legeay A, Gonzalez P, Coppin F, Orjollet D, Camilleri V, Beaugelin-Seiller K, Gilbin R, Simon O. Are antioxidant and transcriptional responses useful for discriminating between chemo- and radiotoxicity of uranium in the crayfish Procambarus clarkii? Ecotoxicol Environ Saf. 2012;80:266-72. https://doi.org/10.1016/j.ecoenv.2012.03.010.

25. Bonvillain CP, Rutherford DA, Kelso WE, Green CC. Physiological biomarkers of hypoxic stress in red swamp crayfish Procambarus clarkii from field and laboratory experiments. Comp Biochem Physiol A Mol Integr Physiol. 2012; 163(1):15-21. https://doi.org/10.1016/j.cbpa.2012.04.015.

26. Du H, Fu L, Xu Y, Kil Z, Xu Z. Improvement in a simple method for isolating white spot syndrome virus (WSSV) from the crayfish Procambarus clarkii. Aquaculture. 2007;262(2-4):532-4. https://doi.org/10.1016/j.aquaculture.2 006.11.002.

27. Ou J, Li Y, Ding Z, Xiu Y, Wu T, Du J, Li W, Zhu H, Ren Q, Gu W, Wang W. Transcriptome-wide identification and characterization of the Procambarus clarkii microRNAs potentially related to immunity against Spiroplasma eriocheiris infection. Fish Shellfish Immunol. 2013:35(2):607-17. https://doi. org/10.1016/j.fsi.2013.05.013.

28. Dong X, Li Z, Wang X, Zhou M, Lin L, Zhou Y, Li J. Characteristics of Vibrio parahaemolyticus isolates obtained from crayfish (Procambarus clarkii) in freshwater. Int J Food Microbiol. 2016;238:132-8. https://doi.org/10.1016/j. ijfoodmicro.2016.09.004.

29. Yi S, Li Y, Shi L, Zhang L. Novel insights into antiviral gene regulation of red swamp crayfish, Procambarus clarkii, infected with white spot syndrome virus. Genes (Basel). 2017;8(11):320. https://doi.org/10.3390/ genes8110320.

30. Du Z, Jin $Y$, Ren D. In-depth comparative transcriptome analysis of intestines of red swamp crayfish, Procambarus clarkii, infected with WSSV. Sci Rep. 2016:6:26780.

31. Calderón-Rosete G, González-Barrios JA, Lara-Lozano M, Piña-Leyva C, Rodríguez-Sosa L. Transcriptional identification of related proteins in the immune system of the crayfish Procambarus clarkii. High Throughput. 2018; 7(3):26. https://doi.org/10.3390/ht7030026.

32. Nian YY, Chen BK, Wang JJ, Zhong WT, Fang Y, Li Z, Zhang QS, Yan DC Transcriptome analysis of Procambarus clarkii infected with infectious hypodermal and haematopoietic necrosis virus. Fish Shellfish Immunol. 2020;98:766-72. https://doi.org/10.1016/j.fsi.2019.11.027.

33. Metzker ML. Sequencing technologies - the next generation. Nat Rev Genet. 2010;11(1):31-46. https://doi.org/10.1038/nrg2626.

34. Ozsolak F, Milos PM. RNA sequencing: advances, challenges and opportunities. Nat Rev Genet. 2011;12(2):87-98. https://doi.org/10.1038/nrg2934.

35. Martin JA, Wang Z. Next-generation transcriptome assembly. Nat Rev Genet. 2011;12(10):671-82. https://doi.org/10.1038/nrg3068.
36. Grabherr MG, Haas BJ, Yassour M, Levin JZ, Thompson DA, Amit I, Adiconis X, Fan L, Raychowdhury R, Zeng Q, Chen Z, Mauceli E, Hacohen N, Gnirke A, Rhind N, di Palma F, Birren BW, Nusbaum C, Lindblad-Toh K, Friedman N, Regev A. Full-length transcriptome assembly from RNA-Seq data without a reference genome. Nat Biotechnol. 2011;29(7):644-52. https://doi.org/10.103 8/nbt.1883.

37. Shen H, Hu Y, Ma Y, Zhou X, Xu Z, Shui Y, Li C, Xu P, Sun X. In-depth transcriptome analysis of the red swamp crayfish Procambarus clarkii. PLoS One. 2014;9(10):e110548. https://doi.org/10.1371/journal.pone.011 0548 .

38. Manfrin C, Tom M, De Moro G, Gerdol M, Giulianini PG, Pallavicini A. The eyestalk transcriptome of red swamp crayfish Procambarus clarkii. Gene. 2015;557(1):28-34. https://doi.org/10.1016/j.gene.2014.12.001.

39. Jiang H, Xing Z, Lu W, Qian Z, Yu H, Li J. Transcriptome analysis of red swamp crawfish Procambarus clarkii reveals genes involved in gonadal development. PLoS One. 2014;9(8):e105122. https://doi.org/10.1371/journal. pone.0105122.

40. Wolin EM, Laufer $H$, Albertini DF. Uptake of the yolk protein, lipovitellin, by developing crustacean oocytes. Dev Biol. 1973;35(1):160-70. https://doi. org/10.1016/0012-1606(73)90013-4.

41. Kulkarni GK, Glade L, Fingerman M. Oogenesis and effects of neuroendocrine tissues on in vitro synthesis of protein by the ovary of the red swamp crayfish Procambarus clarkii (Girard). J Crustacean Biol. 1991; 11(4):513-22. https://doi.org/10.2307/1548520.

42. Laufer $\mathrm{H}$, Biggers WJ, Ahl JS. Stimulation of ovarian maturation in the crayfish Procambarus clarkii by methyl farnesoate. Gen Comp Endocrinol. 1998;111(2):113-8. https://doi.org/10.1006/gcen.1998.7109.

43. Castañon-Cervantes O, Lugo C, Aguilar M, Gonzalez-Moran G, Fanjul-Moles ML. Photoperiodic induction on the growth rate and gonads maturation in the crayfish Procambarus clarkii during ontogeny. Comp Biochem Physiol A. 1995;110(2):139-46. https://doi.org/10.1016/0300-9629(94)00149-N.

44. Rodríguez EM, López Greco LS, Medesani DA, Laufer H, Fingerman M. Effect of methyl farnesoate, alone and in combination with other hormones, on ovarian growth of the red swamp crayfish, Procambarus clarkii, during vitellogenesis. Gen Comp Endocrinol. 2002;125(1):34-40. https://doi.org/10.1 006/gcen.2001.7724

45. Rodríguez EM, Medesani DA, Greco LS, Fingerman M. Effects of some steroids and other compounds on ovarian growth of the red swamp crayfish, Procambarus clarkii, during early vitellogenesis. J Exp Zool. 2002; 292(1):82-7. https://doi.org/10.1002/jez.1144.

46. Mo A, Wang J, Yuan M, Zhao D, Gu Z, Liu Y, Huang H, Yuan YC. Effect of sub-chronic dietary L-selenomethionine exposure on reproductive performance of red swamp crayfish, (Procambarus clarkii). Environ Pollut. 2019;253:749-58. https://doi.org/10.1016/j.envpol.2019.07.082.

47. Silveyra GR, Silveyra P, Vatnick I, Medesani DA, Rodríguez EM. Effects of atrazine on vitellogenesis, steroid levels and lipid peroxidation, in female red swamp crayfish Procambarus clarkii. Aquat Toxicol. 2018;197:136-42. https://doi.org/10.1016/j.aquatox.2018.02.017.

48. Shui Y, Guan ZB, Xu ZH, Zhao CY, Liu DX, Zhou X. Proteomic identification of proteins relevant to ovarian development in the red swamp crayfish Procambarus clarkii. Aquaculture. 2012;370-371:14-8.

49. Kang PF, Mao B, Fan C, Wang YF. Transcriptomic information from the ovaries of red swamp crayfish (Procambarus clarkii) provides new insights into development of ovaries and embryos. Aquaculture. 2019;505:333-43. https://doi.org/10.1016/j.aquaculture.2019.02.074.

50. Bolger AM, Lohse M, Usadel B. Trimmomatic: a flexible trimmer for Illumina sequence data. Bioinformatics. 2014;30(15):2114-20. https://doi.org/10.1093/ bioinformatics/btu170

51. Haas BJ, Papanicolaou A, Yassour M, Grabherr M, Blood PD, Bowden J, Couger MB, Eccles D, Li B, Lieber M, MacManes MD, Ott M, Orvis J, Pochet N, Strozzi F, Weeks N, Westerman R, William T, Dewey CN, Henschel R, LeDuc RD, Friedman N, Regev A. De novo transcript sequence reconstruction from RNA-seq using the trinity platform for reference generation and analysis. Nat Protoc. 2013;8(8):1494-512. https://doi.org/10.1 038/nprot.2013.084.

52. Wang L, Wang S, Li W. RSeQC: quality control of RNA-seq experiments. Bioinformatics. 2012;28(16):2184-5. https://doi.org/10.1093/bioinformatics/ bts356.

53. Tatusov RL, Galperin MY, Natale DA, Koonin EV. The COG database: a tool for genome-scale analysis of protein functions and evolution. Nucleic Acids Res. 2000;28(1):33-6. https://doi.org/10.1093/nar/28.1.33. 
54. Finn RD, Coggill P, Eberhardt RY, Eddy SR, Mistry J, Mitchell AL, Potter SC, Punta M, Qureshi M, Sangrador-Vegas A, Salazar GA, Tate J, Bateman A. The Pfam protein families database: towards a more sustainable future. Nucleic Acids Res. 2016;44(Database issue):D279-85. https://doi.org/10.1093/nar/ gkv1344.

55. Marchler-Bauer A, Zheng C, Chitsaz F, Derbyshire MK, Geer LY, Geer RC, Gonzales NR, Gwadz M, Hurwitz DI, Lanczycki CJ, Lu F, Lu S, Marchler GH, Song JS, Thanki N, Yamashita RA, Zhang D, Bryant SH. CDD: conserved domains and protein three-dimensional structure. Nucleic Acids Res. 2013; 41(Database issue):D348-52. https://doi.org/10.1093/nar/gks1243.

56. Kanehisa M, Goto S. KEGG: Kyoto encyclopedia of genes and genomes. Nucleic Acids Res. 2000;28(1):27-30. https://doi.org/10.1093/nar/28.1.27.

57. Altschul SF, Madden TL, Schäffer AA, Zhang J, Zhang Z, Miller W, Lipman DJ. Gapped BLAST and PSI-BLAST: a new generation of protein database search programs. Nucleic Acids Res. 1997;25(17):3389-402. https://doi.org/10.1093/ nar/25.17.3389.

58. The UniProt Consortium. UniProt: a hub for protein information. Nucleic Acids Res. 2015;43(Database issue):D204-12. https://doi.org/10.1093/nar/ gku989.

59. Moriya Y, Itoh M, Okuda S, Yoshizawa AC, Kanehisa M. KAAS: an automatic genome annotation and pathway reconstruction server. Nucleic Acids Research. 2007;35(Web Server issue):W182-5.

60. Langmead B, Salzberg SL. Fast gapped-read alignment with bowtie 2. Nat Methods. 2012;9(4):357-9. https://doi.org/10.1038/nmeth.1923.

61. Quinlan AR, Hall IM. BEDTools: a flexible suite of utilities for comparing genomic features. Bioinformatics. 2010;26(6):841-2. https://doi.org/10.1093/ bioinformatics/btq033.

62. Patro R, Duggal G, Love MI, Irizarry RA, Kingsford C. Salmon provides fast and bias-aware quantification of transcript expression. Nat Methods. 2017 14(4):417-9. https://doi.org/10.1038/nmeth.4197.

63. Langfelder $P$, Horvath S. WGCNA: an R package for weighted correlation network analysis. BMC Bioinformatics. 2008;9(1):559. https://doi.org/10.11 86/1471-2105-9-559.

64. Love MI, Huber W, Anders S. Moderated estimation of fold change and dispersion for RNA-seq data with DESeq 2. Genome Biol. 2014;15(12):550. https://doi.org/10.1186/s13059-014-0550-8.

65. Szklarczyk D, Franceschini A, Wyder S, Forslund K, Heller D, Huerta-Cepas J, Simonovic M, Roth A, Santos A, Tsafou KP, Kuhn M, Bork $P$, Jensen $L$, von Mering C. STRING v10: protein-protein interaction networks, integrated over the tree of life. Nucleic Acids Res. 2015;43(Database issue):D447-52. https:// doi.org/10.1093/nar/gku1003.

66. Alexa A, Rahnenführer J, Lengauer T. Improved scoring of functional groups from gene expression data by decorrelating GO graph structure. Bioinformatics. 2006;22(13):1600-7. https://doi.org/10.1093/bioinformatics/ btl140.

67. Yu G, Wang LG, Han Y, He QY. clusterProfiler: an R package for comparing biological themes among gene clusters. OMICS. 2012;16(5):284-7. https:// doi.org/10.1089/omi.2011.0118.

68. Meng XL, Liu P, Jia FL, Li J, Gao BQ. De novo Transcriptome analysis of Portunus trituberculatus ovary and testis by RNA-Seq: identification of genes involved in gonadal development. PLoS One. 2015;10(6):e0128659. https:// doi.org/10.1371/journal.pone.0128659.

69. Uawisetwathana U, Leelatanawit R, Klanchui A, Prommoon J, Klinbunga S, Karoonuthaisiri N. Insights into eyestalk ablation mechanism to induce ovarian maturation in the black tiger shrimp. PLoS One. 2011;6(9):e24427. https://doi.org/10.1371/journal.pone.0024427.

70. Liu K, Rajareddy S, Liu L, Jagarlamudi K, Boman K, Selstam G, Reddy P. Control of mammalian oocyte growth and early follicular development by the oocyte PI3 kinase pathway: new roles for an old timer. Dev Biol. 2006; 299(1):1-11. https://doi.org/10.1016/j.ydbio.2006.07.038.

71. Li J, Kawamura K, Cheng Y, Liu S, Klein C, Liu S, Duan EK, Hsueh AJ. Activation of dormant ovarian follicles to generate mature eggs. Proc Natl Acad Sci U S A. 2010;107(22):10280-4. https://doi.org/10.1073/pnas.10011 98107.

72. Grosbois J, Demeestere I. Dynamics of PI3K and hippo signaling pathways during in vitro human follicle activation. Hum Reprod. 2018;33(9):1705-14. https://doi.org/10.1093/humrep/dey250.

73. Lee HN, Chang EM. Primordial follicle activation as new treatment for primary ovarian insufficiency. Clin Exp Reprod Med. 2019;46(2):43-9. https:// doi.org/10.5653/cerm.2019.46.2.43.
74. Dupont J, Reverchon M, Cloix L, Froment P, Ramé C. Involvement of adipokines, AMPK, PI3K and the PPAR signaling pathways in ovarian follicle development and cancer. Int J Dev Biol. 2012;56(10-12):959-67. https://doi. org/10.1387/ijdb.120134jd.

\section{Publisher's Note}

Springer Nature remains neutral with regard to jurisdictional claims in published maps and institutional affiliations.
Ready to submit your research? Choose BMC and benefit from:

- fast, convenient online submission

- thorough peer review by experienced researchers in your field

- rapid publication on acceptance

- support for research data, including large and complex data types

- gold Open Access which fosters wider collaboration and increased citations

- maximum visibility for your research: over $100 \mathrm{M}$ website views per year

At $\mathrm{BMC}$, research is always in progress.

Learn more biomedcentral.com/submissions 